\title{
COMPACTNESS, EXISTENCE AND MULTIPLICITY FOR THE SINGULAR MEAN FIELD PROBLEM WITH SIGN-CHANGING POTENTIALS
}

\author{
FRANCESCA DE MARCHIS, RAFAEL LÓPEZ-SORIANO AND DAVID RUIZ
}

\begin{abstract}
In this paper we consider a mean field problem on a compact surface without boundary in presence of conical singularities. The corresponding equation, named after Liouville, appears in the Gaussian curvature prescription problem in Geometry, and also in the Electroweak Theory and in the abelian Chern-Simons-Higgs model in Physics. Our contribution focuses on the case of sign-changing potentials, and gives results on compactness, existence and multiplicity of solutions.
\end{abstract}

\section{INTRODUCTION}

The classical problem of prescribing the Gaussian curvature on a compact surface $\Sigma$ under a conformal change of the metric dates back to $[12,37]$. Let us denote by $g$ the original metric, $\tilde{g}$ the new one and $e^{v}$ the conformal factor (that is, $\tilde{g}=e^{v} g$ ). The problem reduces to solving the PDE

$$
-\Delta_{g} v+2 K_{g}(x)=2 K(x) e^{v}
$$

where $K_{g}, K$ denote the curvature with respect to $g$ and $\tilde{g}$, respectively. Observe that by the Uniformization Theorem we can assume that $K_{g}$ is a constant. The solvability of this equation has been studied for a long time, and it is not possible to give here a comprehensive list of references.

The above setting needs to be modified if one wants to prescribe also the appearance of conical singularities on the surface, a case that was first studied in [51]. We recall that a conformal metric $\tilde{g}$ has a conical singularity at $p$ of order $\alpha \in(-1,+\infty)$ if there exist local coordinates $z$ in $\mathbb{C}$ such that $z(p)=0$ and

$$
\hat{g}(z)=e^{\psi}|z|^{2 \alpha}|d z|^{2}
$$

where $\hat{g}$ is the local expression of $g$ and $\psi$ is continuous in a neighborhood of 0 in $\mathbb{C}$ and $C^{2}$ outside the point $p$.

In this case we are led with weak solutions of the problem

$$
-\Delta_{g} v+2 K_{g}=2 K(x) e^{v}-4 \pi \sum_{j=1}^{m} \alpha_{j} \delta_{p_{j}}
$$

2010 Mathematics Subject Classification. 35J20, 35R01, 53A30.

Key words and phrases. Prescribed Gaussian curvature problem, conical singularities, variational methods, Morse theory. 
where $\delta_{p_{j}}$ denotes a Dirac delta at the point $p_{j} \in \Sigma$ (see Appendix of [3] for a rigorous deduction of (1.2)). Integrating the above equation and taking into account the Gauss-Bonnet formula, we obtain

$$
4 \pi \chi(\Sigma)=2 \int_{\Sigma} K e^{v} d V_{g}-4 \pi \sum_{j=1}^{m} \alpha_{j}
$$

We now transform equation (1.2) into another one which admits a variational structure. Let $G(x, y)$ be the Green function of the Laplace-Beltrami operator on $\Sigma$ associated to $g$, i.e.

$$
-\Delta_{g} G(x, y)=\delta_{y}-\frac{1}{|\Sigma|} \quad \text { in } \quad \Sigma, \quad \int_{\Sigma} G(x, y) d V_{g}(x)=0
$$

We define

$$
h_{m}(x)=4 \pi \sum_{j=1}^{m} \alpha_{j} G\left(x, p_{j}\right)=2 \sum_{j=1}^{m} \alpha_{j} \log \left(\frac{1}{d\left(x, p_{j}\right)}\right)+2 \pi \alpha_{j} H\left(x, p_{j}\right),
$$

where $H$ is the regular part of $G$. By the change of variable

$$
u=v+h_{m}
$$

we can pass to the equation

$$
-\Delta_{g} u=\lambda\left(\frac{\tilde{K} e^{u}}{\int_{\Sigma} \tilde{K} e^{u} d V_{g}}-\frac{1}{|\Sigma|}\right) \quad \text { in } \quad \Sigma
$$

where

$$
\tilde{K}=K e^{-h_{m}},
$$

and, according to (1.3), $\lambda$ is given by

$$
\lambda=4 \pi\left(\chi(\Sigma)+\sum_{j=1}^{m} \alpha_{j}\right)
$$

Notice that $e^{v}=e^{-h_{m}} e^{u}$ which is consistent with (1.1). Observe also that

$$
\tilde{K}(x) \simeq d\left(x, p_{j}\right)^{2 \alpha_{j}} K(x) \quad \text { close to } p_{j} .
$$

This equation appears also in Physics, in the mathematical Glashow-Salam-Weinberg model of the Electroweak Theory and in the abelian Chern-Simons-Higgs model, see [33,39,49,52]. In this context, the points $p_{j}$ represent vortices of order $\alpha_{j} \in \mathbb{N}$. There are by now many works dealing with this problem, see $[3-7,9,10,17,18,24-26,29,38,45,46,50]$. In this framework, the equation receives the name of (singular) mean field equation. We highlight that under this perspective the restriction (1.7) is not present.

In this paper we are concerned with equation $(*)_{\lambda}$ in the case in which $K$ is a sign-changing function. We give existence and generic multiplicity results by means of variational methods. For that one also needs to show that solutions of $(*)_{\lambda}$ are a priori bounded, which in this case means that they form a compact set. 
Regarding compactness of solutions, one can pose the question as follows: given $u_{n}$ a sequence of solutions of $(*)_{\lambda}$ for $\lambda=\lambda_{n} \rightarrow \lambda_{0}$, is it uniformly bounded? This question has been addressed in [16,41] for the regular problem, and in [8,9] for the equation with vortices, always for positive potentials $K(x)$. In summary, if blow-up occurs then $e^{u_{n}}$ concentrate around a finite set of critical points, and a quantization argument shows that $\lambda_{0}$ must belong to a certain discrete critical set. Here, the assumption on the positivity of $K$ is not just a technical one, as can be inferred from some recent examples of blowing-up solutions in $[13,30]$. Those solutions concentrate around local maxima of $K$ at 0 level.

The question of compactness has been elided in [42] by using energy estimates in a related problem posed on a surface with boundary. This technique is however very much restricted to the case considered there.

For sign-changing functions $K$ the first related compactness result is [20]. That paper is concerned with the scalar curvature prescription problem, a higher dimension analogue of our problem which has also attracted much attention in the literature. The authors are able to show compactness of solutions under the hypothesis

(H1) $K$ is a sign-changing $C^{2, \alpha}$ function with $\nabla K(x) \neq 0$ for any $x \in \Sigma$ with $K(x)=0$.

An improvement of this technique has been given in [23]. The general idea is to first derive uniform integral estimates, which allow one to obtain a priori estimates in the region $\{x \in$ $\Sigma: K(x)<-\delta\}$, for $\delta>0$ small. Then the moving plane technique is used to compare the values of $u$ on both sides of the nodal curve $\Gamma=\{x \in \Sigma: K(x)=0\}$. This, together with the aforementioned integral estimate, implies boundedness in a neighborhood of $\Gamma$. Finally, we rely on $[8,9,41]$ for the region $\{x \in \Sigma: K(x)>\delta\}$.

The approach of [20] has been partially adapted to problem $(*)_{\lambda}$ in $[21,29]$. However, those results use the stereographic projection to pass to a global problem in the plane and are hence restricted to $\Sigma=\mathbb{S}^{2}$. Moreover, the derivation of the integral estimate [21, Lemma 2.2], essential in both papers, is not completely clear. One of the goals of this paper is to settle the question of compactness: we show compactness for $(*)_{\lambda}$ in any compact surface under assumption $(\mathrm{H} 1)$.

Our approach follows the ideas of [23]. The main difficulty with respect to [23] comes from the fact that $u_{n}$ is neither positive nor uniformly bounded from below, a priori. This is a problem for the integral estimate in [21,23], and also for the use of the moving plane method near the nodal curve. In our proofs we first estimate the negative part of $u_{n}$ by using Kato inequality. This is the key for the proof of the integral estimate and is also essential to perform the comparison argument by the moving plane method.

For what concerns existence and multiplicity of solutions, we shall restrict ourselves to the case of positive orders $\alpha_{j}$. Our proofs make use of variational methods. Indeed, problem $(*)_{\lambda}$ is the Euler-Lagrange equation of the energy functional

$$
I_{\lambda}(u)=\frac{1}{2} \int_{\Sigma}|\nabla u|^{2} d V_{g}+\frac{\lambda}{|\Sigma|} \int_{\Sigma} u d V_{g}-\lambda \log \int_{\Sigma} \tilde{K} e^{u} d V_{g}
$$

defined in the domain

$$
X=\left\{u \in H^{1}(\Sigma): \int_{\Sigma} \tilde{K} e^{u} d V_{g}>0\right\} .
$$

If $\lambda<8 \pi$, then $I_{\lambda}$ is coercive and a minimizer exists, see [51]. Instead, $I_{\lambda}$ is not bounded from below if $\lambda>8 \pi$. This range is the main concern of this paper, and we shall use a Morse 
theoretical approach to find solutions of $(*)_{\lambda}$ which are saddle-type critical points of $I_{\lambda}$. In order to do that we study the topology of the energy sublevels of $I_{\lambda}$. In a certain sense, a function $u$ at a low energy level concentrate around a certain number of points of $\Sigma$, and the topology of the space of those configurations plays a crucial role in the min-max argument. This approach has been followed in different works. For instance, [31] considers an analogue problem in dimension 4, whose ideas can be applied equally well to the regular mean field problem (see [32]), whereas [3,18,45] deal with the singular problem. Let us point out that all those papers consider positive functions $K$. For sign changing potentials, the location of the points is restricted to the set $\overline{\{x \in \Sigma: K(x)>0\}}$, and this fact changes dramatically the topology of the space of configurations.

Roughly speaking, to obtain existence of solutions one needs to show that the very low energy sublevels form a non-contractible set. If $\Sigma=\mathbb{S}^{2}$ this study has been carried out in [29], yielding existence of solutions. In this paper we consider the case of general $\Sigma$ and we also prove multiplicity results. For multiplicity, we need to estimate the sum of the Betti numbers of the energy sublevels. This multiplicity result is valid under nondegeneracy assumptions, which are generic (in the couple $(K, g)$ ) by a transversality argument.

In general, min-max arguments yield existence of solutions provided that the well-known Palais-Smale property is satisfied. In this type of problems the validity of that property is still an open problem; however we can circumvent this difficulty by using the deformation lemma established in [43], in the spirit of [48]. This technique has now become well known, and relies on compactness of solutions of approximating problems. At this point our aforementioned compactness result comes into play.

The rest of the paper is organized as follows. In Section 2 we set the notation, recall some preliminary results and state the main theorems proved in the paper. Section 3 is devoted to the proof of the compactness result, see Theorem 2.1. In Section 4 we give a description of the topology of the energy sublevels. The estimation of the dimension of the homology groups of those sets is made in Section 5. Finally, the proofs of our main results are completed in Section 6.

\section{NOTATIONS, MAIN RESULTS AND PRELIMINARIES}

In this section we fix the notation used in this paper, formulate the principal results obtained and collect some preliminary known results.

From now on $(\Sigma, g)$ will be a compact surface without boundary $\Sigma$ equipped with a Riemannian metric $g$ and $d(x, y)$ will denote the distance between two points $x, y \in \Sigma$ induced by the ambient metric. $B_{p}(r)$ stands for the open ball of radius $r>0$ and center $p \in \Sigma$ and

$$
\Omega^{r}=\{x \in \Sigma: d(x, \Omega)<r\} .
$$

Given $f \in L^{1}(\Sigma)$, we denote the mean value of $f$ by $f_{\Sigma} f=\frac{1}{|\Sigma|} \int_{\Sigma} f$, where $|\Sigma|$ is the area of $\Sigma$. Since the functional $I_{\lambda}$ is invariant under addition of constants, we can restrict its domain to functions with 0 mean. In other words, we can consider $I_{\lambda}$ defined in $\bar{X}$, where

$$
\bar{X}=\left\{u \in X: \int_{\Sigma} u d V_{g}=0\right\} .
$$

For a real number $a$, we introduce the following notation for the sublevels of the energy functional $I_{\lambda}$ (defined in (1.8)) restricted to $\bar{X}$

$$
I_{\lambda}^{a}=\left\{u \in \bar{X}: I_{\lambda}(u) \leq a\right\} .
$$


The symbol $\amalg$ will be employed to denote the disjoint union of sets.

Throughout the paper, the sign $\simeq$ refers to homotopy equivalences, while $\cong$ refers to homeomorphisms between topological spaces or isomorphisms between groups.

Given a metric space $M$ and $k \in \mathbb{N}$, we denote by $\operatorname{Bar}_{k}(M)$ the set of formal barycenters of order $k$ on $M$, namely the following family of unit measures supported in at most $k$ points

$$
\operatorname{Bar}_{k}(M)=\left\{\sum_{i=1}^{k} t_{i} \delta_{x_{i}}: t_{i} \in[0,1], \sum_{i=1}^{k} t_{i}=1, x_{i} \in M\right\} .
$$

We consider $\operatorname{Bar}_{k}(M)$ as a topological space with the weak* topology of measures.

Uninfluential positive constants are denoted by $C$, and the value of $C$ is allowed to vary from formula to formula.

\subsection{Main results.}

Let us define the sets

$$
\Sigma^{+}=\{x \in \Sigma: K(x)>0\}, \quad \Sigma^{-}=\{x \in \Sigma: K(x)<0\}, \quad \Gamma=\{x \in \Sigma: K(x)=0\} .
$$

Assumption (H1) implies that the nodal line $\Gamma$ is regular and that

$$
N^{+}=\#\left\{\text { connected components of } \Sigma^{+}\right\}<+\infty .
$$

In what follows we will assume that:

(H2) $p_{j} \notin \Gamma$ for all $j \in\{1, \ldots, m\}$.

So we can suppose, up to reordering, that there exists $\ell \in\{0, \ldots, m\}$ such that

$$
p_{j} \in \Sigma^{+} \text {for } j \in\{1 \ldots, \ell\}, \quad p_{j} \in \Sigma^{-} \text {for } j \in\{\ell+1, \ldots, m\} \text {. }
$$

For $K>0$ it is known that the blow-up phenomena can only occur if the parameter $\lambda$ takes the form $8 \pi r+\sum_{j=1}^{m} 8 \pi\left(1+\alpha_{j}\right) n_{j}$, with $r \in \mathbb{N} \cup\{0\}, n_{j} \in\{0,1\}$ (see [9]). Therefore the set of solutions is compact if $\lambda$ has a different form. In the next theorem we obtain an analogous conclusion without the sign restriction, where the set of critical values is

$$
\Lambda=\left\{8 \pi r+\sum_{j=1}^{\ell} 8 \pi\left(1+\alpha_{j}\right) n_{j}: r \in \mathbb{N} \cup\{0\}, n_{j} \in\{0,1\}\right\} \backslash\{0\} .
$$

Theorem 2.1. Assume that $\alpha_{1}, \ldots, \alpha_{m}>-1$ and let $K_{n}$ be a sequence of functions with $K_{n} \rightarrow K$ in $C^{2, \alpha}$ sense, where $K$ satisfies (H1), (H2). Let $u_{n}$ be a sequence of weak solutions of the problem

$$
-\Delta_{g} u_{n}=\tilde{K}_{n} e^{u_{n}}-f_{n} \text { in } \Sigma,
$$

with $f_{n} \rightarrow f$ in $C^{0, \alpha}$ sense and $\tilde{K}_{n}=K_{n} e^{-h_{m}}$ with $h_{m}$ given by (1.5). Then, the following alternative holds

(1) either $u_{n}$ is uniformly bounded in $L^{\infty}(\Sigma)$;

(2) or, up to a subsequence, $u_{n}$ diverges to $-\infty$ uniformly;

(3) or, up to a subsequence, $\lim _{n \rightarrow+\infty} \int_{\Sigma} \tilde{K}_{n} e^{u_{n}} \in \Lambda$, defined in (2.5). 
We point out that that the conical singularities located in $\Sigma^{-}$do not play any role in the compactness result. Observe also that equation $(*)_{\lambda}$ can be written in the form (2.6) by adding a suitable constant to $u_{n}=u$, if $\tilde{K}_{n}=\tilde{K}$ and $f_{n}=\frac{\lambda}{|\Sigma|}$.

In order to state our existence result we introduce an additional assumption on $K$ :

(H3) $N^{+}>k$ or $\Sigma^{+}$has a connected component which is not simply connected, where $N^{+}$is defined in (2.3).

Theorem 2.2. Let $\alpha_{1}, \ldots, \alpha_{\ell}>0$, where $\ell$ is defined in (2.4), and $\lambda \in(8 k \pi, 8(k+1) \pi) \backslash \Lambda$. If $(H 1)$, (H2), (H3) are satisfied then $(*)_{\lambda}$ admits a solution.

For $K>0$, and thus $\Sigma^{+}=\Sigma$ and $N^{+}=1,(\mathrm{H} 3)$ is satisfied if the surface $\Sigma$ has positive genus; this case has been covered in [3].

If $\Sigma^{+}$has trivial topology Theorem 2.2 is not applicable. We can give a result also in this case, following the ideas of [45]. For that, we define the set:

$$
J_{\lambda}=\left\{p_{j} \in \Sigma^{+}: \lambda<8 \pi\left(1+\alpha_{j}\right)\right\}
$$

and we introduce the hypothesis

(H4) $J_{\lambda} \neq \emptyset$.

Theorem 2.3. Let $\alpha_{1}, \ldots, \alpha_{\ell} \in(0,1]$, where $\ell$ is defined in (2.4), and $\lambda \in(8 \pi, 16 \pi) \backslash \Lambda$. If (H1), (H2), (H4) are satisfied then $(*)_{\lambda}$ admits a solution.

Remark 2.4. There are many examples of applications of these results to the geometric problem commented in the Introduction. Just to exhibit an example, let us consider the problem of prescribing a conformal metric in $\Sigma=\mathbb{T}^{2}$ with gaussian curvature $K$ and one conical point $p$ of order $\alpha$. Assume that assumptions (H1), (H2) are satisfied. Then Theorem 2.2 implies that the problem is solvable if one of the following assumptions are satisfied:

(1) $\alpha \in(2 k, 2 k+2)$ with $k \in \mathbb{N}$ and $\Sigma^{+}$has more than $k$ connected components.

(2) $\alpha \in(2 k, 2 k+2)$ with $k \in \mathbb{N}$ and $\Sigma^{+}$has a component which is not simply connected.

Let us now consider the same problem but with $m$ conical points, all of them of order $\alpha$. Then Theorem 2.3 implies that the geometric problem is solvable if $\alpha \in(0,1]$ and $2<m \alpha<2+2 \alpha$ and at least one conical point is placed in $\Sigma^{+}$.

Many other examples can be constructed.

In order to now state our multiplicity results we introduce some more notation. Let us denote by $A_{i}$ the non-contractible connected components of $\Sigma^{+}$and $C_{h}$ be the contractible ones, $i=$ $1, \ldots, N, h=1, \ldots, M$ and $N, M \in \mathbb{N} \cup\{0\}, N+M=N^{+}$. Obviously,

$$
\Sigma^{+}=\coprod_{i=1}^{N} A_{i} \amalg \coprod_{h=1}^{M} C_{h} .
$$

Recall that a bouquet of $g$ circles is a set $B^{g}=\bigcup_{j=1}^{g} S_{j}^{\prime}$ where $S_{j}^{\prime}$ are simple closed curves verifying that $S_{i}^{\prime} \cap S_{j}^{\prime}=\{q\}$. If $A_{i}$ has genus $g_{i}$ and $b_{i}$ boundary components, it is well known that $A_{i}$ can be retracted to an inner bouquet $B^{g_{i}}$, where $g_{i}=2 g_{i}+b_{i}-1$. Instead, $C_{h}$ is homotopically equivalent to any point $y_{h} \in C_{h}$. Therefore 


$$
\Sigma^{+} \simeq \coprod_{i=1}^{N} B^{g_{i}} \amalg\left\{y_{1}, \ldots, y_{M}\right\}, \quad \text { with } \quad g_{i}=2 g_{i}+b_{i}-1 \quad \text { for } \quad i=1, \ldots, N .
$$

Define $\mathcal{M}$ as the space of all Riemannian metrics on $\Sigma$ equipped with the $\mathcal{C}^{2, \alpha}$ norm and

$$
\mathcal{K}_{\ell}=\left\{K: \Sigma \rightarrow \mathbb{R}: \begin{array}{l}
K \text { satisfies }(\mathrm{H} 1),(\mathrm{H} 2) \\
p_{1}, \ldots, p_{\ell} \in \Sigma^{+}, p_{\ell+1}, \ldots, p_{m} \in \Sigma^{-}
\end{array}\right\},
$$

also equipped with the $C^{2, \alpha}$ norm.

Theorem 2.5. Let $\ell \in\{0, \ldots, m\}$ and let $u$ s assume that $\alpha_{1}, \ldots, \alpha_{\ell}>0$. If $\lambda \in(8 k \pi, 8(k+1) \pi) \backslash \Lambda$, $k \in \mathbb{N}$, then for a generic choice of function $K$ and metric $g$ (namely for $(K, g)$ in an open and dense subset of $\left.\mathcal{K}_{\ell} \times \mathcal{M}\right)$, then

$$
\#\left\{\text { solutions of }(*)_{\lambda}\right\} \geq \sum_{q \geq 0} d_{q},
$$

where if $k+1-M \leq N$, then

$$
d_{q}= \begin{cases}\left(\begin{array}{l}
N+M-1 \\
N+M-p
\end{array}\right) & \sum_{\substack{a_{1}+\ldots+a_{N}=k-p+1 \\
a_{i} \geq 0}} s_{a_{1}, g_{1}} \ldots s_{a_{N}, g_{N}} \\
0 & \text { if } q=2 k-p(1 \leq p \leq k+1),\end{cases}
$$

while if $k+1-M \geq N$, then

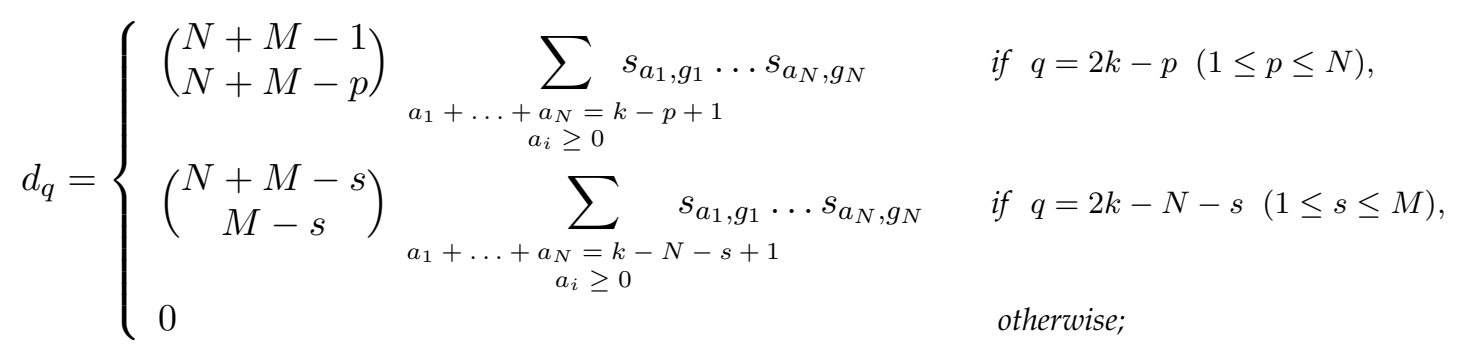

with $s_{a, g}=\left(\begin{array}{c}a+g-1 \\ g-1\end{array}\right)$ and $g_{i}$ defined in (2.8).

Moreover we adopt the following convention: if $N=0$

$$
\sum_{\substack{a_{1}+\ldots+a_{N}=h \\ a_{i} \geq 0}} s_{a_{1}, g_{1}} \ldots s_{a_{N}, g_{N}}= \begin{cases}1 & \text { if } h=0 \\ 0 & \text { if } h \neq 0\end{cases}
$$

Notice that if $k+1-M=N$ the two formulas coincide.

We point out that $\#$ solutions of $\left.(*)_{\lambda}\right\} \rightarrow+\infty$ as $N^{+}=N+M \rightarrow+\infty$.

The above result gives no information if $\Sigma^{+}$has trivial topology; however, our second multiplicity result can be applied also in this case. 
Theorem 2.6. Let $\ell \in\{0, \ldots, m\}$ and let us assume that $\alpha_{1}, \ldots, \alpha_{\ell} \in(0,1]$. If $\lambda \in(8 \pi, 16 \pi) \backslash \Lambda$, then for a generic choice of function $K$ and metric $g$ (namely for $(K, g)$ in an open and dense set of $\mathcal{K}_{\ell} \times \mathcal{M}$ ), then

$$
\#\left\{\text { solutions of }(*)_{\lambda}\right\} \geq N^{+}-1+\sum_{i=1}^{N} g_{i}+\left|J_{\lambda}\right|,
$$

where the set $J_{\lambda}$ is defined in (2.7) and $g_{i}$ in (2.8).

Remark 2.7. We point out that by standard elliptic estimates any solution $u$ of $(*)_{\lambda}$ is classical if all the $\alpha_{j}$ 's are positive, while if $\alpha_{j} \in(-1,0)$ for $j \in J \subset\{1, \ldots, m\}$, then $u \in C^{2}\left(\Sigma \backslash\left\{\cup_{j \in J} p_{j}\right\}\right) \cap C^{0}(\Sigma)$.

\subsection{Topological and Morse-theoretical preliminaries.}

In this subsection we recall a classical theorem on Morse inequalities. Furthermore we give a short review of basic notions of algebraic topology needed to get the multiplicity estimates. Finally, we state a recent result concerning the topology of barycenter sets with disconnected base space.

Given a pair of spaces $(X, A)$ we will denote by $H_{q}\left(X, A ; \mathbb{Z}_{2}\right)$ the relative q-th homology group with coefficient in $\mathbb{Z}_{2}$ and by $\tilde{H}_{q}\left(X ; \mathbb{Z}_{2}\right)=H_{q}\left(X, x_{0} ; \mathbb{Z}_{2}\right)$ the reduced homology with coefficient in $\mathbb{Z}_{2}$, where $x_{0} \in X$. We adopt the convention that $\tilde{H}_{q}\left(X ; \mathbb{Z}_{2}\right)=0$ for any $q<0$.

Finally, if $X, Y$, are two topological spaces and $f: X \rightarrow Y$ is a continuous function, we will denote by $f_{*}: H_{q}\left(X ; \mathbb{Z}_{2}\right) \rightarrow H_{q}\left(Y ; \mathbb{Z}_{2}\right)$ the pushforward morphism induced by $f$.

Let us first recall a classical result in Morse theory.

Theorem 2.8. (see e.g. [19], Theorem 4.3) Suppose that $H$ is a Hilbert manifold, $I \in C^{2}(H ; \mathbb{R})$ satisfies the $(P S)$-condition at any level $c \in[a, b]$, where $a, b$ are regular values for $I$. If all the critical points of $I$ in $\{a \leq I \leq b\}$ are nondegenerate, then

\# $\{$ critical points of $I$ in $\{a \leq I \leq b\}$ with index $q\} \geq \operatorname{dim}\left(H_{q}\left(\{I \leq b\},\{I \leq a\} ; \mathbb{Z}_{2}\right)\right) \quad$ for any $q \geq 0$, where we call (Morse) index of $u \in H$ the number of negative eigenvalues (counted with multiplicity) of the selfadjoint operator $d^{2} I(u)$.

In what follows we collect some well-known definitions and results in algebraic topology and we refer to [36] for further details.

Wedge sum. Given spaces $C$ and $D$ with chosen points $c_{0} \in C$ and $d_{0} \in D$, then the wedge sum $C \vee D$ is the quotient of the disjoint union $C \amalg D$ obtained by identifying $c_{0}$ and $d_{0}$ to a single point. If $\left\{c_{0}\right\}$ (resp. $\left\{d_{0}\right\}$ ) is a closed subspace of $C$ (resp. $D$ ) and is a deformation retract of some neighborhood in $C$ (resp. $D$ ), then

$$
\tilde{H}_{q}\left(C \vee D ; \mathbb{Z}_{2}\right) \cong \tilde{H}_{q}\left(C ; \mathbb{Z}_{2}\right) \bigoplus \tilde{H}_{q}\left(D ; \mathbb{Z}_{2}\right)
$$

see [36, Corollary 2.25].

Smash Product. Inside a product space $X \times Y$ there are copies of $X$ and $Y$, namely $X \times\left\{y_{0}\right\}$ and $\left\{x_{0}\right\} \times Y$ for points $x_{0} \in X$ and $y_{0} \in Y$. These two copies of $X$ and $Y$ in $X \times Y$ intersect 
only at the point $\left(x_{0}, y_{0}\right)$, so their union can be identified with the wedge sum $X \vee Y$. The smash product $X \wedge Y$ is then defined to be the quotient $X \times Y / X \vee Y$. For the reduced homology of the smash product the following formula holds, [36, page 276],

$$
\tilde{H}_{q}\left(C \wedge D ; \mathbb{Z}_{2}\right) \cong \bigoplus_{i+j=q-1}\left(\tilde{H}_{i}\left(C ; \mathbb{Z}_{2}\right) \otimes \tilde{H}_{j}\left(D ; \mathbb{Z}_{2}\right)\right)
$$

Unreduced suspension. The unreduced suspension (often, as in [36], denoted by $S C$ ) is defined to be

$$
\Sigma C=(C \times[0,1]) /\left\{\left(c_{1}, 0\right) \simeq\left(c_{2}, 0\right) \text { and }\left(c_{1}, 1\right) \simeq\left(c_{2}, 1\right) \text { for all } c_{1}, c_{2} \in C\right\} .
$$

For the reduced homology of the unreduced suspension the following formula holds, [36, page 132, ex. 20],

$$
\tilde{H}_{q+1}\left(\Sigma C ; \mathbb{Z}_{2}\right) \cong \tilde{H}_{q}\left(C ; \mathbb{Z}_{2}\right)
$$

Join. The join of two spaces $C$ and $D$ is the space of all segments joining points in $C$ to points in $D$. It is denoted by $C * D$ and is the identification space

$$
C * D=C \times[0,1] \times D /(c, 0, d) \sim\left(c^{\prime}, 0, d\right),(c, 1, d) \sim\left(c, 1, d^{\prime}\right) \quad \forall c, c^{\prime} \in C, \forall d, d^{\prime} \in D .
$$

Being $C * D \simeq \Sigma(C \vee D)$, [36, page 20, ex. 24], we have that

$$
\tilde{H}_{q}\left(C * D ; \mathbb{Z}_{2}\right) \cong \tilde{H}_{q}\left(\Sigma(C \vee D) ; \mathbb{Z}_{2}\right)
$$

At last, we present a recent result obtained in [1, Theorem 5.19] concerning the space of formal barycenters on a disjoint union of spaces.

Proposition 2.9. For $C, D$ two disjoint connected spaces and $k \geq 2, \operatorname{Bar}_{k}(C \amalg D)$ has the homology of

$$
\begin{aligned}
& \operatorname{Bar}_{k}(C) \vee \Sigma \operatorname{Bar}_{k-1}(C) \vee \operatorname{Bar}_{k}(D) \vee \Sigma \operatorname{Bar}_{k-1}(D) \vee \\
& \vee \bigvee_{\ell=1}^{k-1}\left(\operatorname{Bar}_{k-\ell}(C) * \operatorname{Bar}_{\ell}(D)\right) \vee \bigvee_{\ell=2}^{k-1}\left(\Sigma \operatorname{Bar}_{k-\ell}(C)\right) * \operatorname{Bar}_{\ell-1}(D)
\end{aligned}
$$

\section{COMPACTNESS OF SOLUTIONS}

In this section we present the proof of Theorem 2.1, a compactness result for solutions $u_{n}$ of the general problem (2.6).

As commented previously, most of the results in this direction consider only the case of positive $K$, like for instance $[16,41]$ for the regular case and $[8,9]$ for the singular one. In order to prove Theorem 2.1, and following [20], we will first derive an a priori estimate in the region in which $K$ is negative, and later we will give such estimates in the nodal region of $K$.

Proposition 3.1. Given $\delta>0$, there exists $C>0$ such that $u_{n}(x) \leq C$ for all $x \in \Sigma^{-} \backslash \Gamma^{\delta}, n \in \mathbb{N}$.

Proposition 3.2. There exist $\varepsilon, C>0$, such that $u_{n}(x) \leq C$ for all $n \in \mathbb{N}$ and $x \in \Gamma^{\varepsilon}$. 
The proof of Theorem 2.1 will be finally accomplished by studying the possible blow-up of the sequence $u_{n}$ in $\Sigma^{+} \backslash \Gamma^{\varepsilon}$.

One of the difficulties in our study is that we do not know a priori whether the term

$$
\int_{\Sigma}\left|\tilde{K}_{n}\right| e^{u_{n}} d V_{g}
$$

is bounded or not. By standard regularity results, this would give a priori $W^{1, p}$ estimates $(p \in(1,2))$ on $u_{n}$. Instead, if we integrate (2.6) we only obtain that $\int_{\Sigma} \tilde{K}_{n} e^{u_{n}}$ is bounded.

Our first lemma shows that such kind of estimate is indeed possible for $u_{n}^{-}=\min \left\{u_{n}, 0\right\}$. This fact will be useful to prove both Propositions 3.1 and 3.2.

Lemma 3.3. Under the conditions of Theorem 2.1, define $v_{n}=u_{n}^{-}-f_{\Sigma} u_{n}^{-}$. Then there exists $C>0$ such that

a) $\left\|v_{n}\right\|_{L^{p}} \leq C$ for any $p \in[1,+\infty)$;

b) $v_{n}(x) \geq-C$ for any $x \in \Sigma$.

Proof. We apply the well-known Kato inequality to the operator $\Delta_{g}$ (see for instance [2, Theorem 5.1])

$$
-\Delta_{g} u_{n}^{-} \geq\left(-\Delta_{g} u_{n}\right) \chi_{\left\{u_{n} \leq 0\right\}}=\left(\tilde{K}_{n} e^{u_{n}}-f_{n}\right) \chi_{\left\{u_{n} \leq 0\right\}} \geq-C h(x),
$$

where

$$
h(x)=1+\sum_{\substack{j \geq \ell+1 \\ \alpha_{j}<0}} d\left(x, p_{j}\right)^{2 \alpha_{j}} .
$$

Observe that $h \in L^{q}(\Sigma)$ for $q \in[1,1+\delta)$ if $\delta>0$ is sufficiently small.

Since the Radon measures $\mu_{n}=-\Delta_{g} u_{n}^{-} \geq-C h(x)$ are given as a divergence (in the sense of distributions), then $\int_{\Sigma} d \mu_{n}=0$. From that we conclude that $\int_{\Sigma} d\left|\mu_{n}\right|$ is bounded. We use the Green's representation for $v_{n}$ and Hölder inequality to obtain:

$$
\left|v_{n}(x)\right|^{p} \leq\left(\int_{\Sigma} d\left|\mu_{n}\right|\right)^{p-1} \int_{\Sigma}|G(x, y)|^{p} d\left|\mu_{n}(y)\right|
$$

We now integrate in $x$ and make use of Fubini Theorem, taking into account that the Green function of $\Delta_{g}$ in $\Sigma$ belongs to $L^{p}$ :

$$
\begin{array}{r}
\left\|v_{n}\right\|_{L^{p}}^{p} \leq\left(\int_{\Sigma} d\left|\mu_{n}\right|\right)^{p-1} \int_{\Sigma} \int_{\Sigma}|G(x, y)|^{p} d\left|\mu_{n}(y)\right| d x \\
=\left(\int_{\Sigma} d\left|\mu_{n}\right|\right)^{p-1} \int_{\Sigma} \int_{\Sigma}|G(x, y)|^{p} d x d\left|\mu_{n}(y)\right| \leq C\left(\int_{\Sigma} d\left|\mu_{n}\right|\right)^{p} .
\end{array}
$$

This concludes the proof of a).

For the proof of b), we write the Green function of $\Delta_{g}$ in $\Sigma$ as $G(x, y)=-\frac{1}{2 \pi} \log (r d(x, y))+$ $\tilde{H}(x, y)$, where $\tilde{H}: \Sigma \times \Sigma \rightarrow \mathbb{R}$ is a bounded function. Here we have chosen $r \in\left(0, \operatorname{diam}(\Sigma)^{-1}\right)$. Then, 


$$
\begin{aligned}
v_{n}(x)= & \int_{\Sigma} G(x, y) d \mu_{n}(y)=-\frac{1}{2 \pi} \int_{\Sigma} \log (r d(x, y)) d \mu_{n}^{+}(y) \\
& -\frac{1}{2 \pi} \int_{\Sigma} \log (r d(x, y)) d \mu_{n}^{-}(y)+\int_{\Sigma} \tilde{H}(x, y) d \mu_{n}(y) .
\end{aligned}
$$

By the choice of $r>0$,

$$
-\frac{1}{2 \pi} \int_{\Sigma} \log (r d(x, y)) d \mu_{n}^{+}(y) \geq 0
$$

Moreover, by (3.2),

$$
\left.-\frac{1}{2 \pi} \int_{\Sigma} \log (r d(x, y)) d \mu_{n}^{-}(y) \geq-C \frac{1}{2 \pi} \int_{\Sigma} \log (r d(x, y)) h(y)\right) d y \geq-C,
$$

and finally

$$
\left|\int_{\Sigma} \tilde{H}(x, y) d \mu_{n}(y)\right| \leq\|\tilde{H}\|_{L^{\infty}} \int_{\Sigma} d\left|\mu_{n}\right| \leq C
$$

As a first consequence of Lemma 3.3, we present an integral estimate in domains entirely contained in the positive or negative region. This result is an extension of the Chen-Li integral estimate for positive solutions, see [23]. In our case $u_{n}$ may change sign, but we can perform the estimate thanks to Lemma 3.3.

Lemma 3.4. Under the conditions of Theorem 2.1, for every open subdomain $\Sigma_{0}$ completely contained in $\Sigma^{+}$or $\Sigma^{-}$, there exists $C>0$ so that

$$
\left|\int_{\Sigma_{0}} \tilde{K}_{n} e^{u_{n}} d V_{g}\right| \leq C
$$

Proof. Take $\Sigma_{1}$ a smooth domain such that $\overline{\Sigma_{0}} \subset \Sigma_{1} \subset \overline{\Sigma_{1}} \subset \Sigma^{ \pm}$. Let $\varphi$ be the first eigenfunction of the Laplace operator in $\Sigma_{1}$, that is,

$$
\begin{cases}-\Delta_{g} \varphi=\lambda_{1} \varphi & \text { in } \Sigma_{1} \\ \varphi=0 & \text { on } \partial \Sigma_{1}\end{cases}
$$

Next, we multiply (2.6) by $\varphi^{2}$, and integrate by parts over $\Sigma_{1}$ to obtain

$$
\int_{\Sigma_{1}} \tilde{K}_{n} \varphi^{2} e^{u_{n}}=-\int_{\Sigma_{1}} u_{n} \Delta_{g}\left(\varphi^{2}\right)+O(1)
$$

Let us denote $f=\Delta_{g}\left(\varphi^{2}\right)=2\left(|\nabla \varphi|^{2}-\lambda_{1} \varphi^{2}\right)$. Observe that $\int_{\Sigma_{1}} f=0$. Then

$$
\int_{\Sigma_{1}} u_{n}^{-} f=\int_{\Sigma_{1}}\left(u_{n}^{-}-f_{\Sigma} u_{n}^{-}\right) f
$$

so that, by Lemma 3.3, a),

$$
\left|\int_{\Sigma_{1}} u_{n}^{-} f\right| \leq\left\|u_{n}^{-}-f_{\Sigma} u_{n}^{-}\right\|_{L^{1}\left(\Sigma_{1}\right)}\|f\|_{L^{\infty}\left(\Sigma_{1}\right)} \leq C .
$$


On the other hand, for any $\gamma>0$,

$$
\int_{\Sigma_{1}} u_{n}^{+}|f| \leq C \int_{\Sigma_{1}} u_{n}^{+} \leq C \int_{\Sigma_{1}} u_{n}^{+} \frac{\left|\varphi^{2} \tilde{K}_{n}\right|^{\gamma}}{\left|\varphi^{2} \tilde{K}_{n}\right|^{\gamma}}
$$

By Young inequality we obtain

$$
\int_{\Sigma_{1}} u_{n}^{+}|f| \leq \varepsilon \int_{\Sigma_{1}}\left|u_{n}^{+}\right|^{\frac{1}{\gamma}} \varphi^{2}\left|\tilde{K}_{n}\right|+C_{\varepsilon} \int_{\Sigma_{1}} \frac{1}{\left|\varphi^{2} \tilde{K}_{n}\right|^{\frac{\gamma}{1-\gamma}}}
$$

We can take $\gamma>0$ sufficiently small so that the second integral term in the right hand side is finite (recall that, by Hopf lemma, $\varphi \sim d\left(x, \partial \Sigma_{1}\right)$ near the boundary). Then, by (3.4), (3.5) and (3.6)

$$
\int_{\Sigma_{1}}\left|\tilde{K}_{n}\right| \varphi^{2} e^{u_{n}} \leq C+\varepsilon \int_{\Sigma_{1}}\left|u_{n}^{+}\right|^{\frac{1}{\gamma}} \varphi^{2}\left|\tilde{K}_{n}\right|
$$

We now use the inequality $\left(t^{+}\right)^{\frac{1}{\gamma}} \leq C+e^{t}$ to conclude that

$$
\int_{\Sigma_{1}}\left|\tilde{K}_{n}\right| \varphi^{2} e^{u_{n}} \leq C
$$

finishing the proof.

In order to prove Proposition 3.1, we will need the following result, which is based on a mean value inequality for subharmonic functions.

Lemma 3.5. Let $w$ be a function defined in $\Sigma_{0} \subset \Sigma, x_{0} \in \Sigma_{0}$, and assume that $-\Delta_{g} w(x) \leq A$ for all $x \in \Sigma_{0}$, for some positive value $A>0$. Take $R>0$ such that

$$
R<\min \left\{\frac{1}{5} d\left(x_{0}, \partial \Sigma_{0}\right), \frac{1}{2} \operatorname{diam}\left(\Sigma_{0}\right)\right\} .
$$

Then there exists $C>0$ depending only on $\Sigma_{0}$ and $A$ such that

$$
\sup _{x \in B_{x_{0}}(R / 4)} w(x) \leq C\left(1+f_{B_{x_{0}}(R)} w\right) .
$$

Proof. Define $v$ as the solution of the problem

$$
\begin{cases}-\Delta_{g} v=-A, & \text { in } \Sigma_{0} \\ v=0, & \text { on } \partial \Sigma_{0}\end{cases}
$$

Clearly $v$ is smooth and $w+v$ is a subharmonic function. We now apply the mean value inequality for subharmonic functions (see [40, Theorem 2.1] for its version on manifolds) to conclude. 
Proof of Proposition 3.1. Take $\Sigma_{0} \subset \overline{\Sigma_{0}} \subset \Sigma^{-}, x \in \Sigma_{0}$ and fix $r>0$ sufficiently small. We apply Lemma 3.5 to $w=u^{+}$and we obtain

$$
\begin{gathered}
\sup _{B_{x}(r)} u_{n}^{+}(x) \leq C+C \int_{B_{x}(4 r)} u_{n}^{+}=C+C \int_{B_{x}(4 r)} \frac{u_{n}^{+}}{p} \frac{\left(-\tilde{K}_{n}\right)^{1 / p}(x)}{\left(-\tilde{K}_{n}\right)^{1 / p}(x)} \\
\leq C+C \int_{B_{x}(4 r)} e^{\frac{u_{n}}{p}} \frac{\left(-\tilde{K}_{n}\right)^{1 / p}(x)}{\left(-\tilde{K}_{n}\right)^{1 / p}(x)} \leq C+C\left(\int_{B_{x}(4 r)}-\tilde{K}_{n}(x) e^{u_{n}}\right)^{1 / p}\left(\int_{B_{x}(4 r)} \frac{1}{\left(-\tilde{K}_{n}\right)^{\frac{1}{p-1}}(x)}\right)^{\frac{p-1}{p}} .
\end{gathered}
$$

It suffices to choose a large enough $p$ and use Lemma 3.4 to conclude that $\sup _{B_{x}(R)} u_{n}^{+}(x)<C$.

We now turn our attention to Proposition 3.2. The proof follows the argument of [23], with the main difference that our solutions $u_{n}$ are not positive. This difficulty can be bypassed thanks to the following lemma, whose proof is based on Lemma 3.3.

Lemma 3.6. Under the hypotheses of Theorem 2.1, and given $\delta>0$, there exists $C>0$ such that

$$
u_{n}\left(x_{0}\right)-u_{n}\left(x_{1}\right) \leq C
$$

for every $n \in \mathbb{N}, x_{0} \in \Sigma^{-} \backslash \Gamma^{\delta}, x_{1} \in \Sigma$. Moreover, for any $r_{0}>0$, there exists $C>0$ such that

$$
\left|\nabla u_{n}(x)\right| \leq C \quad \forall x \in \Sigma^{-} \backslash\left(\Gamma^{\delta} \cup \bigcup_{i=\ell+1}^{m} B_{p_{i}}\left(r_{0}\right)\right) .
$$

Proof. By Lemma 3.3, b), we have that

$$
u_{n}\left(x_{1}\right)-f_{\Sigma} u_{n}^{-} \geq u_{n}^{-}\left(x_{1}\right)-f_{\Sigma} u_{n}^{-} \geq C .
$$

Taking into account Lemma 3.5, we have that for any fixed $r \in\left(0, \frac{\delta}{2}\right)$,

$$
u_{n}\left(x_{0}\right)-f_{\Sigma} u_{n}^{-} \leq C\left(1+f_{B_{x_{0}}(r)}\left(u_{n}(x)-f_{\Sigma} u_{n}^{-}\right)\right)
$$

Moreover, by Proposition 3.1, $u_{n}(x) \leq u_{n}^{-}(x)+C$ for all $x \in B_{x_{0}}(r)$. Making use of Lemma 3.3, a), we conclude

$$
u_{n}\left(x_{0}\right)-f_{\Sigma} u_{n}^{-} \leq C\left(1+f_{B_{x_{0}}(r)}\left|u_{n}^{-}(x)-f_{\Sigma} u_{n}^{-}\right|\right) \leq C
$$

This estimate, together with (3.9), shows that (3.7) holds true.

We now turn our attention to the proof of (3.8). Given $r_{0}>0$, take any $p>2$ and fix $x$ such that $B_{x}(r) \subset \Sigma^{-} \backslash\left(\Gamma^{\delta} \cup \bigcup_{i=\ell+1}^{m} B_{p_{i}}\left(r_{0}\right)\right)$. Recall the inequality (see [35, Theorem 9.11])

$$
\left\|u_{n}-f_{\Sigma} u_{n}^{-}\right\|_{W^{2, p}\left(B_{x}\left(\frac{r}{2}\right)\right)} \leq C\left(\left\|\tilde{K}_{n} e^{u_{n}}-f_{n}\right\|_{L^{p}\left(B_{x}(r)\right)}+\left\|u_{n}-f_{\Sigma} u_{n}^{-}\right\|_{L^{p}\left(B_{x}(r)\right)}\right) .
$$


Combining (3.10) and Lemma 3.3, b), $u_{n}-f_{\Sigma} u_{n}^{-}$is uniformly bounded in $L^{\infty}\left(B_{x}(r)\right)$, whereas Proposition 3.1 implies that $\tilde{K}_{n} e^{u_{n}}-f_{n}$ is uniformly bounded on $B_{x}(r)$. Therefore,

$$
\left\|u_{n}-f_{\Sigma} u_{n}^{-}\right\|_{W^{2, p}\left(B_{x}\left(\frac{r}{2}\right)\right)} \leq C .
$$

In particular (3.8) holds.

Proof of Proposition 3.2. Since the proof is of local nature, we first pass to a problem in a planar domain. Given a point $p \in \Gamma$, we take a small neighborhood $U$ of $\mathrm{p}$ and an isothermal coordinate system $y=\left(y_{1}, y_{2}\right)$ centered at $p$ such that the metric $g$ takes the form $g=e^{\varphi}\left(d y_{1}^{2}+d y_{2}^{2}\right)$ in $\Omega^{\prime} \subset \mathbb{R}^{2}$, where $\varphi$ is smooth and $\varphi(0)=0$. Consequently, for a subdomain $\Omega \subset \Omega^{\prime}, u_{n}$ satisfies

$$
-\Delta u_{n}=e^{\varphi(y)} \tilde{K}_{n}(y) e^{u_{n}}-e^{\varphi(y)} f_{n}(y), \quad \text { in } \Omega
$$

where $\Delta$ is the usual laplacian.

Let us define $u_{0, n}$ as the unique solution for the problem

$$
\begin{cases}\Delta u_{0, n}=e^{\varphi} f_{n}, & \text { in } \Omega \\ u_{0, n}=0, & \text { on } \partial \Omega .\end{cases}
$$

If we now write the equation in the new variable $u_{n}-u_{0, n}$, which will be denoted again by $u_{n}$, we obtain:

$$
-\Delta u_{n}=W_{n}(y) e^{u_{n}} \quad \text { in } \Omega,
$$

where $W_{n}(y)=e^{\varphi(y)} e^{u_{0, n}(y)} \tilde{K}_{n}(y)$.

Moreover, $W_{n} \rightarrow W$ in $C^{2, \alpha}(\bar{\Omega})$. Assumption (H1) is translated to $W$ in the form

$W$ is a $C^{2, \alpha}(\Omega)$ function, changes sign and $\nabla W(x) \neq 0$ in $\Gamma=\{x \in \Omega: W(x)=0\}$.

Our proof is based on the method of moving planes, which allows us to compare the values of $u_{n}$ close to $\Gamma$. For the sake of clarity, we drop the subindex $n$ in the notation of the rest of this proof.

By the assumptions on $W$ for small $\delta>0$, there exists $\beta>0$ s.t.

$$
|\nabla W(y)| \geq \beta \text { for any } y \text { with }|W(y)| \leq \delta .
$$

For a given point $x_{0} \in \Gamma$, take a ball $B \subset\{y \in \Omega: W(y)>0\}$ tangent to $\Gamma$ at $x_{0}$. Applying the Kelvin transform which leaves $\partial B$ invariant and taking a neighborhood of $x_{0}$, we obtain a set (renamed as $\Gamma$ ) which is strictly convex (recall that $\Gamma$ is a $C^{2, \alpha}$ curve).

Next, through a translation and a rotation we define the new system as $x=\left(x_{1}, x_{2}\right)$ and $x_{1}=\gamma\left(x_{2}\right)$ which corresponds to the curve $\Gamma$. Let $\Omega_{\varepsilon}=\left\{x_{1}<\gamma\left(x_{2}\right)+\varepsilon\right\} \cap\left\{x_{1}>-2 \varepsilon\right\}$ and $\partial_{l} \Omega_{\varepsilon}=\left\{x: x_{1}-\gamma\left(x_{2}\right)=\varepsilon\right\}$. In summary, it is possible to choose a conformal map such that, for some $\varepsilon>0$ small, the following hold (see figure):

(i) $x_{0}$ becomes the origin;

(ii) $\Omega_{\varepsilon}$ is located to the left of the line $x_{1}=\varepsilon$ and it is tangent to it;

(iii) $\partial_{l} \Omega_{\varepsilon}$ is uniformly convex; 
(iv) $\frac{\partial W}{\partial x_{1}} \leq-\frac{1}{2} \beta$, for every $x \in \Omega_{\varepsilon}$;

(v) $\overline{\Omega_{\varepsilon}} \cap\left\{p_{1}, \ldots, p_{m}\right\}=\emptyset$.

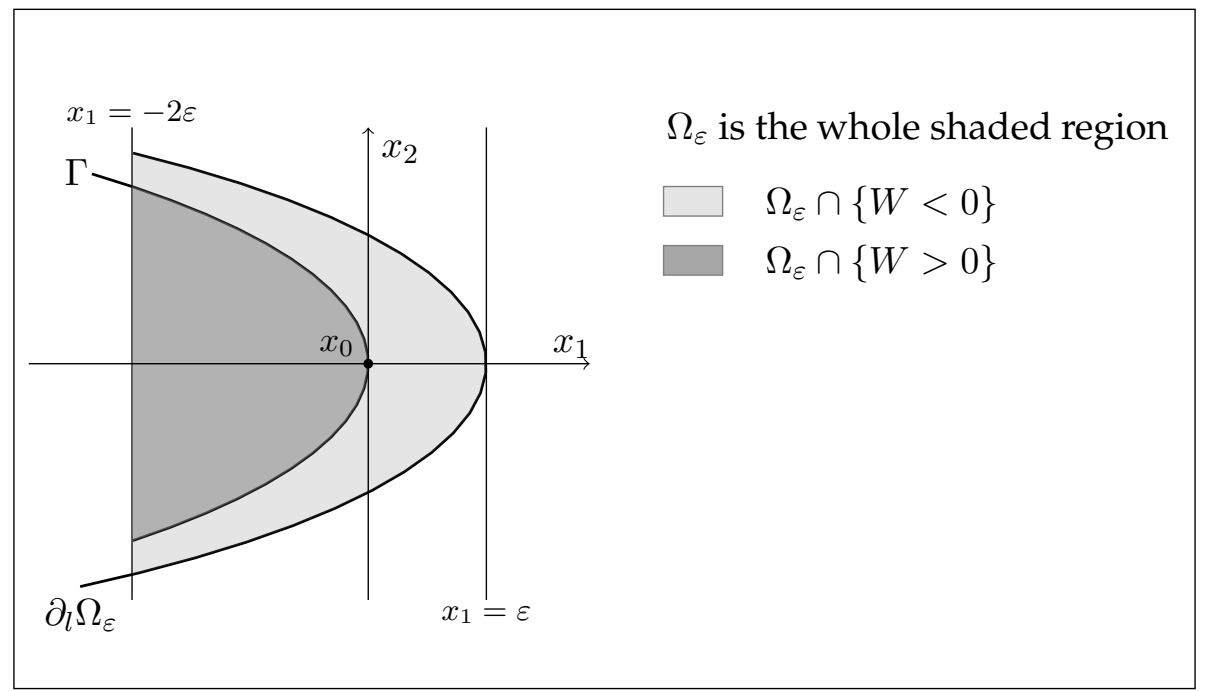

Let $m=\min _{x \in \partial_{l} \Omega_{\varepsilon}} u(x)$ and $M=\max _{x \in \partial_{l} \Omega_{\varepsilon}} u(x)$. We define $\tilde{u} \in C^{2}\left(\overline{\Omega_{\varepsilon}}\right)$ such that:

a) $\tilde{u}=u$ in $\partial_{l} \Omega_{\varepsilon}$;

b) $m \leq \tilde{u} \leq M$;

c) $|\nabla \tilde{u}| \leq C$ in $\Omega_{\varepsilon}$.

Observe that c) is possible by (3.8).

Let $w$ be the harmonic function

$$
\begin{cases}\Delta w=0, & \text { in } \Omega_{\varepsilon} \\ w=\tilde{u}, & \text { on } \partial \Omega_{\varepsilon} .\end{cases}
$$

Due to (3.7), the oscillation of $u$ on $\partial_{l} \Omega_{\varepsilon}$ is bounded, i.e.,

$$
M-m=\max _{\partial_{l} \Omega_{\varepsilon}} u-\min _{\partial_{l} \Omega_{\varepsilon}} u \leq C .
$$

Consequently, the oscillation of $w$ is also bounded in $\Omega_{\varepsilon}$. We also define a new auxiliary function $v$ as

$$
v(x)=u(x)-w(x)+C_{0}\left(\varepsilon+\gamma\left(x_{2}\right)-x_{1}\right),
$$

for some $C_{0}>0$ to be determined. It is clear that the function $v$ verifies

$$
\Delta v+f(x, v(x))-C_{0} \gamma^{\prime \prime}\left(x_{2}\right)=0, \quad \text { in } \Omega_{\varepsilon},
$$

with

$$
f(x, v(x))=W(x) e^{v(x)+w(x)-C_{0}\left(\varepsilon+\gamma\left(x_{2}\right)-x_{1}\right)} .
$$

We claim that for a suitable $C_{0}$ 


$$
v(x) \geq 0 \text { in } \Omega_{\varepsilon} \quad \text { and } \quad v(x)=0 \text { on } \partial_{l} \Omega_{\varepsilon} .
$$

The boundary condition is direct. In order to prove the first part, we distinguish two cases:

- Case 1: $\frac{\varepsilon}{2}<x_{1}-\gamma\left(x_{2}\right) \leq \varepsilon$

Taking into account (v), by (3.8) we have that

Consequently,

$$
\left|\frac{\partial u}{\partial x_{1}}\right| \leq C \text { and }\left|\frac{\partial w}{\partial x_{1}}\right| \leq C
$$

$$
\frac{\partial v}{\partial x_{1}}=\frac{\partial u}{\partial x_{1}}-\frac{\partial w}{\partial x_{1}}-C_{0} \leq C-C_{0} .
$$

It suffices to choose $C_{0}$ sufficiently large to obtain that $\frac{\partial v}{\partial x_{1}}$ is negative. Since $v=0$ on $\partial_{l} \Omega_{\varepsilon}$, it is clear that (3.18) holds.

- Case 2: $x_{1}-\gamma\left(x_{2}\right) \leq \frac{\varepsilon}{2}$ and $x_{1} \geq-2 \varepsilon$

By (3.7), we have that

$$
v(x)=u(x)-w(x)+C_{0}\left(\varepsilon+\gamma\left(x_{2}\right)-x_{1}\right) \geq \min _{\Omega_{\varepsilon}} u-\max _{\partial_{l} \Omega_{\varepsilon}} u+C_{0} \frac{\varepsilon}{2} \geq-C+C_{0} \frac{\varepsilon}{2} .
$$

So, choosing $C_{0}$ sufficiently large, (3.18) holds.

Now we are ready to apply the method of moving planes to $v$ in the $x_{1}$ direction. Thus, we start from $x_{1}=\varepsilon$ and move the line perpendicular to $x_{1}$-axis towards the left. Namely, let $T_{\lambda}=$ $\left\{x \in \mathbb{R}^{2}: x_{1} \geq \lambda\right\}$ the half-plane, $M_{\lambda}=\left\{x \in \mathbb{R}^{2}: x_{1}=\lambda\right\}$ its boundary and $x_{\lambda}=\left(2 \lambda-x_{1}, x_{2}\right)$ the reflection point of $x$ with respect to the line $M_{\lambda}$. Our goal is to prove that

$$
v\left(x_{\lambda}\right) \geq v(x),
$$

for every $x \in T_{\lambda} \cap \Omega_{\varepsilon}$ for $\lambda \in\left[\frac{\varepsilon}{2}-\varepsilon_{1}, \varepsilon\right]$, with some $\varepsilon_{1} \in(0, \varepsilon)$ to be determined. By (3.18) and (3.19), (3.20) holds for $\lambda \in\left(\frac{3}{4} \varepsilon, \varepsilon\right]$.

By a standard argument (see [34]), we see that the moving planes argument can be carried on provided that

$$
f(x, v) \leq f\left(x_{\lambda}, v\right) \quad \text { for every } x \in \Omega_{\varepsilon} \quad \text { with } \quad \varepsilon>\lambda>\frac{\varepsilon}{2}-\varepsilon_{1} .
$$

It is easy to check that (3.21) holds if

$$
\frac{\partial f(x, v(x))}{\partial x_{1}}=e^{u}\left(\frac{\partial W}{\partial x_{1}}+W\left(\frac{\partial w}{\partial x_{1}}+C_{0}\right)\right) \leq 0, \quad \text { if } x \in \Omega_{\varepsilon}, x_{1}>-\varepsilon_{1} .
$$

In other words, if $f$ is monotone decreasing along the direction $(1,0)$, near $x_{0}$.

If $W(x) \leq 0$, it is enough to choose $C_{0}>-\frac{\partial w}{\partial x_{1}}$ to verify

$$
\frac{\partial W}{\partial x_{1}}+W\left(\frac{\partial w}{\partial x_{1}}+C_{0}\right) \leq W\left(\frac{\partial w}{\partial x_{1}}+C_{0}\right) \leq 0 .
$$


In the case that $W(x)>0$ by the assumptions on $W$, for every $\varepsilon_{1}>0$ there exists a neighborhood $V_{\varepsilon_{1}}$ of $\Gamma$ such that $W(x) \leq \varepsilon_{1}$.

Since $\frac{\partial w}{\partial x_{1}}+C_{0}$ is bounded from above, then

$$
\frac{\partial W}{\partial x_{1}}+W\left(\frac{\partial w}{\partial x_{1}}+C_{0}\right) \leq-\frac{\beta}{2}+\varepsilon_{1}\left(\frac{\partial w}{\partial x_{1}}+C_{0}\right) \leq-\frac{\beta}{2}+\varepsilon_{1} C,
$$

therefore we can take $\varepsilon_{1}$ small enough to obtain the desired conclusion. We choose $\varepsilon_{1}<\varepsilon$.

In this way, the method of moving planes works up to $\lambda=\frac{\varepsilon}{2}-\varepsilon_{1}$. Therefore, (3.20) implies that $v(x)$ is monotone decreasing in the $(1,0)$-direction. In fact, we can repeat the previous argument rotating the $x_{1}$-axis by a small angle.

Thus, there exist $\varepsilon_{2}>0$ and a fixed cone $\Delta_{0}$ such that for any $x \in B_{x_{0}}\left(\varepsilon_{2}\right)$ we have

and

$$
v(y) \geq v(x), \forall y \in \Delta_{x}
$$

$$
\emptyset \neq \Delta_{x} \cap \Sigma^{+} \subset \Omega_{\varepsilon}
$$

where $\Delta_{x}$ denotes a translation of the cone $\Delta_{0}$ with $x$ at its vertex. By (3.16), we can transform the previous inequality into

$$
u(y)+C\left(\varepsilon_{1}\right) \geq u(x) \quad \forall y \in \Delta_{x} .
$$

Moreover, there exists $\eta>0$, such that for any $x \in B_{x_{0}}\left(\varepsilon_{2}\right)$ the intersection of the cone $\Delta_{x}$ with the set $\Sigma^{+} \backslash \Gamma^{\eta}$ has a positive measure, and the lower bound of the measure depends only on $\eta$ and the $C^{1}$ norm of $K$. Namely, setting $\Sigma_{x}=\Delta_{x} \cap\left(\Sigma^{+} \backslash \Gamma^{\eta}\right)$ we have that for any $x \in B_{x_{0}}\left(\varepsilon_{2}\right)$

$$
\left|\Sigma_{x}\right| \geq \eta_{1}>0
$$

Thanks to this, the proof can be concluded combining (3.24) and the integral bound of Lemma 3.4. Indeed by virtue of (3.23) and property (v)

$$
\min _{\Sigma_{x}} \tilde{K} \geq \eta_{2}>0
$$

hence

$$
e^{u(x)}=f_{\Sigma_{x}} e^{u(x)} d y \stackrel{(3.24)}{\leq} C f_{\Sigma_{x}} e^{u(y)} d y \stackrel{(3.26)}{\leq} C f_{\Sigma_{x}} \tilde{K} e^{u(y)} d y \stackrel{\text { Lemma } 3.4}{\leq} \frac{C}{\left|\Sigma_{x}\right|} \stackrel{(3.25)}{\leq} C .
$$

Proof of Theorem 2.1. Take $\varepsilon>\delta>0$ and the open set $\Sigma_{1}=\Sigma^{+} \backslash \overline{\Gamma^{\delta}}$, where $\varepsilon$ is given by Proposition 3.2. By Propositions 3.1 and 3.2, $u_{n}$ is uniformly bounded from above in $\Sigma \backslash \Sigma_{1}$. Moreover, by Lemma 3.4, $\int_{\Sigma_{1}} \tilde{K}_{n} e^{u_{n}}$ is bounded. By [9] there are two possibilities:

Case 1: $u_{n}$ is bounded from above in $\Sigma$. Therefore, $\tilde{K}_{n} e^{u_{n}}-f_{n} \in L^{p}\left(\Sigma \backslash \Sigma_{1}\right)$ for some $p>1$. Elliptic regularity estimates imply that $u_{n}-f_{\Sigma} u_{n} \in W^{2, p}(\Sigma)$, so $u_{n}-f_{\Sigma} u_{n} \in L^{\infty}(\Sigma)$. If $f_{\Sigma} u_{n}$ is bounded, we obtain (1); if, on the contrary, a subsequence of $f_{\Sigma} u_{n}$ diverges negatively, we obtain (2). 
Case 2: The sequence $u_{n}$ is not bounded from above. Applying the results of [9] concerning the blow-up analysis for (2.6) in $\Sigma_{1}$, we can assume that there exists a finite blow-up set $S=\left\{q_{1}, \ldots, q_{r}\right\} \subset \Sigma_{1}$. Moreover, by enlarging $\delta$ if necessary, we can assume that $u_{n} \rightarrow-\infty$ uniformly in $\partial \Sigma_{1}$, and

$$
\tilde{K}_{n} e^{u_{n}}-\sum_{i=1}^{r} \beta\left(q_{i}\right) \delta_{q_{i}} \quad \text { in the sense of weak convergence of measures in } \overline{\Sigma_{1}},
$$

with $\beta\left(q_{i}\right) \geq 8 \pi$.

Now, let us define $v$ the solution of the problem

$$
\begin{cases}-\Delta_{g} v=C_{1} h & \text { in } \Sigma \backslash \Sigma_{1} \\ v=0 & \text { on } \partial \Sigma_{1}\end{cases}
$$

where $h$ is defined in (3.3) and $C_{1}$ is a positive constant such that $C_{1} h$ is an uniform upper bound of the term $\tilde{K}_{n} e^{u_{n}}-f_{n}$ in $\Sigma \backslash \Sigma_{1}$. Since $h \in L^{p}\left(\Sigma \backslash \Sigma_{1}\right)$ for some $p>1$, then $v \in L^{\infty}\left(\Sigma \backslash \Sigma_{1}\right)$ by standard regularity results. By the maximum principle, for any $C>0$ there exists $n_{0} \in \mathbb{N}$ such that $u_{n} \leq v-C$ in $\Sigma \backslash \Sigma_{1}$ for $n \geq n_{0}$. This implies that $u_{n} \rightarrow-\infty$ uniformly in $\Sigma \backslash \Sigma_{1}$; in particular,

$$
\tilde{K}_{n} e^{u_{n}} \rightarrow \sum_{i=1}^{r} \beta\left(q_{i}\right) \delta_{q_{i}} \quad \text { in the sense of weak convergence of measures in } \Sigma .
$$

It is worth to point out that, at this point of the proof, we can not apply yet the quantization part of the concentration-compactness Theorem of [9] unless we check the bounded oscillation condition on $\partial \Sigma_{1}$.

By (3.27), employing the Green's representation formula for $u_{n}$, we have that

$$
u_{n}-\overline{u_{n}} \rightarrow \sum_{i=1}^{r} \beta\left(q_{j}\right) G\left(x, q_{j}\right)+h_{m},
$$

uniformly on compact sets of $\Sigma \backslash\left(S \cup\left\{p_{1}, \ldots, p_{\ell}\right\}\right)$, where $h_{m}$ is defined in (1.5). Therefore, the sequence $u_{n}-\overline{u_{n}}$ admits uniformly bounded oscillation on any compact set of $\Sigma \backslash(S \cup$ $\left.\left\{p_{1}, \ldots, p_{\ell}\right\}\right)$. Indeed, there exists a constant $C>0$ such that

$$
\max _{\partial \Sigma_{1}} u_{n}-\min _{\partial \Sigma_{1}} u_{n}<C .
$$

By virtue of this condition, we can apply the quantization result of [9] to conclude that, up to subsequence,

$$
\lim _{k \rightarrow+\infty} \int_{\Sigma} \tilde{K}_{n} e^{u_{n}} \in \Lambda
$$

\section{TOPOLOGICAL DESCRIPTION OF THE ENERGY SUBLEVELS AND MORSE INEQUALITIES}

In this section we study the topology of the energy sublevels of $I_{\lambda}$. We shall observe a change in the topology of the sublevels between high and low ones. This fact will be decisive to prove the existence and multiplicity theorems. 
First, we define a continuous projection $\Psi$ from low sublevels of $I_{\lambda}$ onto a compact topological space, whose character is inherited from the geometry of the function $K$. By using ideas from [45] we give a more accurate description for $k=1$ depending on the order of the conical points.

Next, we define the reverse map $\varphi$ from the corresponding space onto $I_{\lambda}^{-L}=\{u \in \bar{X}$ : $\left.I_{\lambda}(u) \leq-L\right\}$ with $L>0$ large enough such that the composition $\Psi \circ \varphi$ is homotopically equivalent to the identity map.

Finally, we adapt the well-known Morse inequalities for $I_{\lambda}$ which will be crucial to prove the multiplicity theorems.

Since this scheme has been used several times, see [27, 29,31, 45], we will be sketchy and focus on the main differences concerning the sign-changing case.

\subsection{Topological description of the low sublevels of $I_{\lambda}$.}

The first result allows us to project continuously functions $u$ with a low energy level onto the set of formal barycenters on a union of bouquets and a simplex contained in $\Sigma^{+}$.

Proposition 4.1. Let $\lambda \in(8 k \pi, 8 \pi(k+1)), k \in \mathbb{N}$, and assume (H1), (H2). Then for $L>0$ sufficiently large there exists a continuous projection

$$
\Psi: I_{\lambda}^{-L} \longrightarrow \operatorname{Bar}_{k}\left(Z_{N, M}\right)
$$

where

$$
Z_{N, M}=\coprod_{i=1}^{N} B^{g_{i}} \amalg Y_{M} \subset \Sigma^{+} \backslash\left\{p_{1}, \cdots, p_{\ell}\right\} \quad \text { and } \quad Y_{M}=\left\{y_{1}, \ldots, y_{M}\right\},
$$

where $B^{g_{i}} \subset A_{i}$ is a bouquet of $g_{i}$ circles, with $g_{i}$ defined in (2.8), and $y_{h} \in C_{h}$.

Moreover, if $\frac{\tilde{K}^{+} e^{u_{n}}}{\int_{\Sigma} \tilde{K}^{+} e^{u_{n}} d V_{g}} \rightarrow \sigma$, for some $\sigma \in \operatorname{Bar}_{k}\left(Z_{N, M}\right)$, then $\Psi\left(u_{n}\right) \rightarrow \sigma$.

Remark 4.2. Under assumption (H3), the topological set $B_{a} r_{k}\left(Z_{N, M}\right)$ is not contractible. In case $N=0, \operatorname{Bar}_{k}\left(Z_{N, M}\right)$ is the $(k-1)$-skeleton of $(M-1)$-symplex, which is not contractible if $k<M$ (see Exercise 16 in Section 2.2 of [36]).

Proof of Proposition 4.1. This lemma is proved in the spirit of [31], but following closely the approach of [11].

Claim: If $I_{\lambda}\left(u_{n}\right) \rightarrow-\infty$, up to a subsequence,

$$
\sigma_{n}:=\frac{\tilde{K}^{+} e^{u_{n}}}{\int_{\Sigma} \tilde{K}^{+} e^{u_{n}} d V_{g}} \rightarrow \sigma \in \operatorname{Bar}_{k}\left(\overline{\Sigma^{+}}\right) .
$$

Suppose by contradiction that there exist $k+1$ points $x_{1}, \ldots, x_{k+1} \subset \operatorname{supp}(\sigma)$. Take $r>0$ such that $B_{x_{i}}(2 r) \cap B_{x_{j}}(2 r)=\emptyset$ for $i \neq j$. Therefore, there exists $\varepsilon>0$ such that $\sigma\left(B_{x_{i}}(2 r)\right)>2 \varepsilon$. As a consequence, $\sigma_{n}\left(B_{x_{i}}(r)\right) \geq \varepsilon$; this implies that for any $\tilde{\varepsilon}>0$ :

$$
\log \int_{\Sigma} \tilde{K}^{+} e^{u_{n}}-\frac{1}{|\Sigma|} \int_{\Sigma} u_{n} \leq \frac{1}{(16(k+1) \pi-\tilde{\varepsilon})} \int_{\Sigma}\left|\nabla u_{n}\right|^{2} d V_{g}+C,
$$

where $C$ is a positive constant which depends on $\varepsilon, r, \tilde{\varepsilon}$. This kind of improvements of the Moser-Trudinger inequality were first obtained by Chen and Li in [22]. We also refer to [45, 
Proposition 2.2 and Proposition 2.3], where a formulation of this inequality for nonnegative functions was given in the case $k=2$ (the case $k>2$ is analogous).

Taking $\tilde{\varepsilon}$ sufficiently small, (4.2) violates the hypothesis that $I_{\lambda}\left(u_{n}\right)$ diverges negatively, yielding a contradiction.

By the claim, given a neighborhood $V$ of $\operatorname{Bar}_{k}\left(\Sigma^{+}\right)$in the weak topology of measures, there exists $L_{0}>O$ large enough such that if $L>L_{0}$, then

$$
\frac{\tilde{K}^{+} e^{u}}{\int_{\Sigma} \tilde{K}^{+} e^{u} d V_{g}} \in V, \quad \forall u \in I_{\lambda}^{-L} .
$$

In the appendix of [11], it is proved that $\operatorname{Bar}_{k}\left(\overline{\Sigma^{+}}\right)$is a Euclidean Neighborhood Retract. Observe that the weak topology of measures is metrizable on bounded sets, see [15, Theorem 3.28]. By [14, Lemma E.1], there exists $V$ a neighborhood of $\operatorname{Bar}_{k}\left(\overline{\Sigma^{+}}\right)$in the weak topology of measures, and a continuous retraction $\mathcal{X}: V \rightarrow \operatorname{Bar}_{k}\left(\overline{\Sigma^{+}}\right)$. Next, by (4.3), we define $\tilde{\Psi}$ as

$$
\begin{array}{rllll}
\tilde{\Psi}: \quad I_{\lambda}^{-L} & \longrightarrow & V & \stackrel{\mathcal{X}}{\longrightarrow} & \operatorname{Bar}_{k}\left(\overline{\Sigma^{+}}\right) \\
u & \longmapsto & \frac{\tilde{K}^{+} e^{u}}{\int_{\Sigma} \tilde{K}^{+} e^{u} d V_{g}} & \longmapsto & \sum_{i=1}^{k} t_{i} \delta_{x_{i}} .
\end{array}
$$

Observe that we can retract continuously $\overline{A_{i}}$ onto $B^{g_{i}}$ and $\overline{C_{h}}$ onto a single point $y_{h} \in C_{h}$. Consequently, we can define the retraction

$$
r: \overline{\Sigma^{+}} \longrightarrow Z_{N, M}
$$

We are now in conditions to define the map $\Psi$ as the composition of $\tilde{\Psi}$ with the function $r *: \operatorname{Bar}_{k}\left(\overline{\Sigma^{+}}\right) \longrightarrow \operatorname{Bar}_{k}\left(Z_{N, M}\right)$, the pushforward induced by the function $r$, then

$$
\begin{aligned}
\Psi: I_{\lambda}^{-L} & \longrightarrow \operatorname{Bar}_{k}\left(Z_{N, M}\right) \\
u & \longmapsto \sum_{i} s_{i} \delta_{x_{i}},
\end{aligned}
$$

where the values $s_{i}$ are given by $\tilde{\Psi}$. Since $r$ is a retraction, if $\frac{\tilde{K}^{+} e^{u_{n}}}{\int_{\Sigma} \tilde{K}^{+} e^{u_{n}} d V_{g}} \rightarrow \sigma$, for some $\sigma \in$ $\operatorname{Bar}_{k}\left(Z_{N, M}\right)$, then $\Psi\left(u_{n}\right) \rightarrow \sigma$.

On the other hand, for $\lambda \in(8 k \pi, 8 \pi(k+1)), k \in \mathbb{N}$, and $Z$ a compact subset of $\Sigma^{+} \backslash\left\{p_{1}, \ldots, p_{\ell}\right\}$ we consider test functions concentrated in at most $k$ points of $Z$ with arbitrary low energy. For $\gamma>0$ small enough, we consider a smooth nondecreasing cut-off function $\chi_{\gamma}: \mathbb{R}^{+} \rightarrow \mathbb{R}^{+}$such that

$$
\chi_{\gamma}(t)= \begin{cases}t & \text { for } t \in[0, \gamma] \\ 2 \gamma & \text { for } t \geq 2 \gamma\end{cases}
$$

For $\mu>0$ and $\sigma=\sum_{i=1}^{k} t_{i} \delta_{x_{i}} \in \operatorname{Bar}_{k}(Z)$, we define

$$
\begin{gathered}
\phi_{\mu, \sigma}: \Sigma \rightarrow \mathbb{R} \quad \phi_{\mu, \sigma}(x)=\log \sum t_{i}\left(\frac{\mu}{1+\left(\mu \chi_{\gamma}\left(d\left(x, x_{i}\right)\right)\right)^{2}}\right)^{2}, \\
\varphi_{\mu, \sigma}(x)=\phi_{\mu, \sigma}(x)-\int_{\Sigma} \phi_{\mu, \sigma} d V_{g} .
\end{gathered}
$$


By the results in [29, Lemma 4.11, Lemma 4.12], which hold independently of the genus of the surface, we have that

Lemma 4.3. Let $\lambda \in(8 k \pi, 8(k+1) \pi), k \in \mathbb{N}$, and let $Z$ be a compact subset of $\Sigma^{+} \backslash\left\{p_{1}, \ldots, p_{\ell}\right\}$. Then we can choose $\gamma>0$ such that:

(i) given $L>0$ there exists a large $\mu(L)>0$ satisfying that for $\mu \geq \mu(L), \varphi_{\mu, \sigma} \in \bar{X}$, where $\bar{X}$ is defined in (2.1), and $I_{\lambda}\left(\varphi_{\mu, \sigma}\right)<-L$ for any $\sigma \in \operatorname{Bar}_{k}(Z)$;

(ii) for any $\sigma \in \operatorname{Bar}_{k}(Z)$

$$
\frac{\tilde{K}^{+} e^{\varphi_{\mu, \sigma}}}{\int_{\Sigma} \tilde{K}^{+} e^{\varphi_{\mu, \sigma}} d V_{g}} \rightarrow \sigma \quad \text { as } \mu \rightarrow+\infty
$$

The following two results allow us to deal with the case in which $\Sigma^{+}$has only simply connected components and $N^{+} \leq k$; however, it is restricted to $\lambda \in(8 \pi, 16 \pi)$.

Proposition 4.4. Let $\lambda \in(8 \pi, 16 \pi)$ and assume (H1), (H2). Let

$$
J_{\lambda, A_{i}}=J_{\lambda} \cap A_{i} \quad \text { for } i=1, \ldots, N, \quad J_{\lambda, C_{h}}=J_{\lambda} \cap C_{h} \quad \text { for } h=1, \ldots, M,
$$

where $J_{\lambda}$ is defined in (2.7), and let us assume that, up to reordering, $M_{\lambda} \in\{1, \ldots, M\}$ is such that $J_{\lambda, C_{h}} \neq \emptyset$ if $h \in\left\{1, \ldots, M_{\lambda}\right\}$ and $J_{\lambda, C_{h}}=\emptyset$ if $h \in\left\{M_{\lambda}+1, \ldots, M\right\}$.

Then for $L>0$ sufficiently large there exists a continuous projection

$$
\Psi: I_{\lambda}^{-L} \rightarrow \operatorname{Bar}_{1}\left(W_{N, M, J_{\lambda}}\right)
$$

where

$$
W_{N, M, J_{\lambda}}=\coprod_{i=1}^{N} B^{g_{i}+\left|J_{\lambda, A_{i}}\right|} \amalg \coprod_{h=1}^{M_{\lambda}} B^{\left|J_{\lambda, C_{h}}\right|} \amalg \hat{Y}_{M_{\lambda}},
$$

$B^{g_{i}+\left|J_{\lambda, A_{i}}\right|} \subset A_{i}, B^{\left|J_{\lambda, C_{h}}\right|} \subset C_{h}$ are bouquets of $g_{i}+\left|J_{\lambda, A_{i}}\right|$ and $\left|J_{\lambda, C_{h}}\right|$ circles respectively, with $g_{i}$ defined in (2.8), for $i=1, \ldots, N$ and $h=1, \ldots, M_{\lambda}$, and $\hat{Y}_{M_{\lambda}}=\coprod_{h=M_{\lambda}+1}^{M}\left\{y_{h}\right\}$ with $y_{h} \in Y_{h}$ for $h=M_{\lambda}+1, \ldots, M$.

Moreover, if

$$
\frac{\tilde{K}^{+} e^{u_{n}}}{\int_{\Sigma} \tilde{K}^{+} e^{u_{n}} d V_{g}} \rightarrow \sigma, \quad \text { for some } \sigma \in \operatorname{Bar}_{1}\left(W_{N, M, J_{\lambda}}\right)
$$

then $\Psi\left(u_{n}\right) \rightarrow \sigma$.

Remark 4.5. Observe that $W_{N, M, J_{\lambda}}$ is not contractible if and only if either $N \geq 1$ or $M_{\lambda}>1$, i.e. if (H3) holds, or if $J_{\lambda} \neq \emptyset$, namely (H4) holds.

Proof. For $L>0$ sufficiently large and $r>0$, we apply some results of [29]; more specifically, by Propositions 4.4., 4.7., 4.8., and Remark 4.10, of [29], we construct the continuous projection

$$
\beta: I_{\lambda}^{-L} \rightarrow \overline{\Sigma^{+}} \backslash \bigcup_{p_{i} \in J_{\lambda}} B_{p_{i}}(r)
$$


with the property that if $\frac{\tilde{K}^{+} e^{u_{n}}}{\int_{\Sigma} \tilde{K}^{+} e^{u_{n}} d V_{g}} \rightarrow \delta_{x}$ for some $x \in \overline{\Sigma^{+}} \backslash \bigcup_{p_{i} \in J_{\lambda}} B_{p_{i}}(r)$ then $\beta\left(u_{n}\right) \rightarrow x$. Notice that $I_{\lambda}$ is bounded from below on the functions belonging to $\tilde{\Psi}^{-1}\left(J_{\lambda}\right)$.

We can rewrite $\overline{\Sigma^{+}} \backslash \bigcup_{p_{i} \in J_{\lambda}} B_{p_{i}}(r)$ as

$$
\coprod_{i=1}^{N} A_{i}^{\prime} \amalg \coprod_{h=1}^{M_{\lambda}} C_{h}^{\prime} \amalg \coprod_{h=M_{\lambda}+1}^{M} C_{h} .
$$

where $A_{i}^{\prime}=A_{i} \backslash \bigcup_{p_{i} \in J_{\lambda, A_{i}}} B_{p_{i}}(r)$ and $C_{h}^{\prime}=C_{h} \backslash \bigcup_{p_{i} \in J_{\lambda, C_{h}}} B_{p_{i}}(r)$.

The sets $A_{i}^{\prime}$ can be retracted to an inner bouquet $B^{g_{i}+\left|J_{\lambda, A_{i}}\right|} \subset A_{i}^{\prime}$ and $C_{h}^{\prime}$ to $B^{\left|J_{\lambda, C_{h}}\right|} \subset C_{h}^{\prime}$, in a similar way to the proof of Proposition 4.1, we can define a retraction

$$
r: \overline{\Sigma^{+}} \backslash \bigcup_{p_{i} \in J_{\lambda}} B_{p_{i}}(r) \longrightarrow W_{N, M, J_{\lambda}}
$$

Finally, we can define $\Psi$ as the composition of $\beta$ with the pushforward $r *: \operatorname{Bar}_{1}\left(\overline{\Sigma^{+}}\right)$ $\left.\bigcup_{p_{i} \in J_{\lambda}} B_{p_{i}}(r)\right) \longrightarrow \operatorname{Bar}_{1}\left(W_{N, M, J_{\lambda}}\right)$, then

$$
\begin{array}{ccc}
\Psi: I_{\lambda}^{-L} & \longrightarrow & \operatorname{Bar}_{1}\left(W_{N, M, J_{\lambda}}\right) \\
u & \longmapsto & \delta_{x} .
\end{array}
$$

Since $r$ is a retraction, the second part of the proposition is proved.

Next, for $\lambda \in(8 \pi, 16 \pi)$, we introduce appropriate test functions that will allow to map a compact subset $W$ of $\Sigma^{+} \backslash J_{\lambda}$ into low sublevels of $I_{\lambda}$.

Let $\tilde{\alpha}=\max _{n \leq \ell \mid p_{n} \notin J_{\lambda}} \alpha_{n}$ or $\tilde{\alpha}=0$ if $J_{\lambda}=\left\{p_{1}, \ldots, p_{\ell}\right\}$ or $\ell=0$. For any $\alpha \in\left(\tilde{\alpha}, \frac{\lambda}{8 \pi}-1\right), \mu>0$ and $p \in W$, we define

$$
\begin{gathered}
\phi_{\mu, p, \alpha}: \Sigma \rightarrow \mathbb{R}, \quad \phi_{\mu, p, \alpha}(x)=2 \log \left(\frac{\mu^{1+\alpha}}{1+\left(\mu \chi_{\gamma}(d(x, p))\right)^{2(1+\alpha)}}\right), \\
\varphi_{\mu, p, \alpha}(x)=\phi_{\mu, p, \alpha}(x)-\int_{\Sigma} \phi_{\mu, p, \alpha} d V_{g} .
\end{gathered}
$$

where $\chi_{\gamma}$ is defined in (4.5).

Since $W$ is a compact subset of $\Sigma^{+} \backslash J_{\lambda}$ the results in [29, Lemma 4.13, Lemma 4.14] holds, namely

Lemma 4.6. Let $\lambda \in(8 \pi, 16 \pi)$ and let $W$ be a compact subset of $\Sigma^{+} \backslash J_{\lambda}$. Then we can choose $\gamma>0$ such that:

(i) given any $L>0$, there exists a large $\mu(L)>0$ satisfying that, for any $\mu \geq \mu(L), \varphi_{\mu, p, \alpha} \in \bar{X}$ and $I_{\lambda}\left(\varphi_{\mu, p, \alpha}\right)<-L$ for any $p \in W$

(ii) for any $p \in W$,

$$
\frac{\tilde{K}^{+} e^{\varphi_{\mu, p, \alpha}}}{\int_{\Sigma} \tilde{K}^{+} e^{\varphi_{\mu, p, \alpha}} d V_{g}} \rightarrow \delta_{p} \quad \text { as } \mu \rightarrow+\infty
$$




\subsection{Topological characterization of the high sublevels of $I_{\lambda}$.}

The compactness result Theorem 2.1, combined with the deformation Lemma [43, Proposition 2.3], allows us to prove the next alternative bypassing the Palais-Smale condition, which is not known for the functional $I_{\lambda}$.

Lemma 4.7. Let $\lambda \notin \Lambda$ and assume (H1), (H2). If $I_{\lambda}$ has no critical levels inside some interval $[a, b]$, then $I_{\lambda}^{a}$ is a deformation retract of $I_{\lambda}^{b}$.

Remark 4.8. Actually the deformation lemma in [43] is originally proved for the regular case and for $K$ positive, but it adapts in a straightforward way to the singular one, even for $K$ sign-changing.

Indeed, in the proof of Proposition 2.3 of [43] a certain deformation is used following a flow in the domain of the functional, and $I_{\lambda}$ decreases along that flow. In our case $I_{\lambda}$ is not defined in the whole Sobolev space but on $X$, but $I_{\lambda}(u) \rightarrow+\infty$ as u approaches the boundary of $\bar{X}$, so that $\bar{X}$ is positively invariant under this flow. Hence Proposition 2.3 of [43] is applicable and gives Lemma 4.7.

In turn, since Theorem 2.1 implies that the functional $I_{\lambda}$ stays uniformly bounded on the solutions of $(*)_{\lambda}$, the above Lemma can be used to show that it is possible to retract the whole space $\bar{X}$ onto a high sublevel $I_{\lambda}^{b}$ (see [44, Corollary 2.8], also for this issue minor changes are required).

Lemma 4.9. Let $\lambda \notin \Lambda$ and assume (H1), (H2). If $b>0$ is sufficiently large, the sublevel $I_{\lambda}^{b}$ is a retract of $\bar{X}$ and hence is contractible.

\subsection{Morse inequalities for $I_{\lambda}$.}

The aim of this subsection is to prove a Morse-theoretical result for $I_{\lambda}$, which will be crucial to get the multiplicity estimates of Theorem 2.5 and Theorem 2.6.

Proposition 4.10. Let $\ell \in\{0, \ldots, m\}$ and let us assume $\alpha_{1}, \ldots, \alpha_{\ell}>0$. If $\lambda \in(8 \pi,+\infty) \backslash \Lambda$, then for a generic choice of the function $K, g$ (namely for $(K, g)$ in an open and dense subset of $\mathcal{K}_{\ell} \times \mathcal{M}$ ) there exists $b=b(K, g)>0$ such that

- $I_{\lambda}^{b} \equiv I_{\lambda, K, g}^{b}$ is a retract of $\bar{X} \equiv \bar{X}_{K, g}$,

- any solution $u \in I_{\lambda, K, g}^{b}$ of $(*)_{\lambda}$ is nondegenerate,

where to emphasize the dependence on $K$ and $g$ we write

and

$$
\bar{X}_{K, g}=\left\{u \in H_{g}^{1}(\Sigma): \int_{\Sigma} u d V_{g}=0, \int_{\Sigma} K e^{-h_{m}} e^{u} d V_{g}>0\right\}
$$

$$
I_{\lambda, K, g}^{b}=\left\{u \in \bar{X}_{K, g}: \frac{1}{2} \int_{\Sigma}|\nabla u|^{2} d V_{g}-\lambda \log \int_{\Sigma} K e^{-h_{m}} e^{u} d V_{g} \leq b\right\}
$$

with $h_{m}$ defined in (1.5).

Proof. Let us fix $(\bar{K}, \bar{g}) \in \mathcal{K}_{\ell} \times \mathcal{M}$.

Next, we introduce the Banach space $\mathcal{S}$ of all $C^{2, \alpha}$ symmetric matrices on $\Sigma$. The set $\mathcal{M}$ of all $C^{2, \alpha}$ Riemannian metrics on $\Sigma$ is an open subset of $\mathcal{S}$.

It is easy to verify that for small $\delta>0$, and any $g \in \mathcal{G}_{\delta}:=\left\{g \in \mathcal{S}:\|g\|_{C^{2, \alpha}}<\delta\right\}, \bar{g}+g$ is a Riemannian metric and the sets $H_{\bar{g}+g}^{1}(\Sigma), L_{\bar{g}+g}^{2}(\Sigma), L_{\bar{g}+g}^{1}(\Sigma)$ coincide respectively with $H_{g}^{1}(\Sigma)$, $L_{g}^{2}(\Sigma), L_{g}^{1}(\Sigma)$ and the two norms are equivalent.

Being $\bar{K} \in \mathcal{K}_{\ell}$, it satisfies (H1), (H2). Thus, it is not hard to see that for $\delta>0$ small enough $\bar{K}+K$ satisfies (H1), (H2) for any $K \in \mathcal{H}_{\delta}:=\left\{h \in C^{2, \alpha}(\Sigma):\|h\|_{C^{2, \alpha}(\Sigma)}<\delta\right\}$. 
Furthermore, by Theorem 2.1, it suffices to take a smaller $\delta>0$ so that there exists $R>0$ such that for any $(K, g) \in \mathcal{H}_{\delta} \times \mathcal{G}_{\delta}$ all the critical points (with zero mean value) of $I_{\lambda, \bar{K}+K, \bar{g}+g}$ are contained in the ball $B_{0}(R) \subset H_{\bar{g}}^{1}(\Sigma)$.

Taking a smaller $\delta>0$, if necessary, we have by Lemma 4.9 and Theorem 2.1 that there exists $b>0$ such that the sublevel $I_{\lambda, \bar{K}+K, \bar{g}+g}^{b}$ is a retract of $\bar{X}_{\bar{K}+K, \bar{g}+g}$ for any $(K, g) \in \mathcal{H}_{\delta} \times \mathcal{G}_{\delta}$.

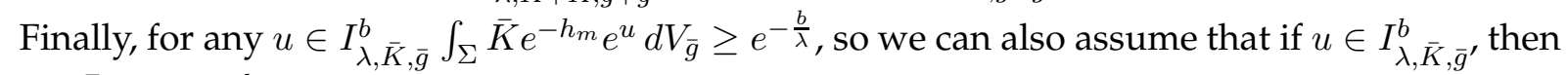
$\int_{\Sigma}(\bar{K}+K) e^{-h_{m}} e^{u} d V_{\bar{g}+g}>0$ for any $(K, g) \in \mathcal{H}_{\delta} \times \mathcal{G}_{\delta}$.

Once $\delta$ is fixed in this way it is possible to argue as in [28], where a transversality Theorem, obtained in [47], is applied to deduce that the following set is an open and dense subset of $\mathcal{H}_{\delta} \times \mathcal{G}_{\delta}$

$$
\left\{(K, g) \in \mathcal{H}_{\delta} \times \mathcal{G}_{\delta}: \begin{array}{l}
\text { any } u \in I_{\lambda, \bar{K}+K, \bar{g}+g}^{b} \text { solution of the equation } \\
-\Delta_{\bar{g}+g} u=\lambda\left(\frac{(\bar{K}+K) e^{-h_{m}} e^{u}}{\int_{\Sigma}(K+K) e^{-h_{m}} e^{u} d V_{\bar{g}+g}}-\frac{1}{\int_{\Sigma} d V_{\bar{g}+g}}\right) \text { is nondegenerate }
\end{array}\right\} .
$$

Since this holds for any choice of $(\bar{K}, \bar{g})$ the thesis follows.

As recalled in the previous subsection we do not know whether $I_{\lambda}$ satisfies the $(P S)$ condition or not, thus Theorem 2.8 can not be directly applied. However, as already pointed out in [3], the $(P S)$-condition can be replaced by the request that appropriate deformation lemmas hold for the functional.

In particular a flow defined by Malchiodi in [44] allows to adapt to $I_{\lambda}$ the classical deformation lemmas [19, Lemma 3.2 and Theorem 3.2] needed so that Theorem 2.8 can be applied for $H=\bar{X}$ and $I=I_{\lambda}$. It is worth to point out that, even if the flow is defined for $K$ positive, arguing as in Remark 4.8 it is not hard to check that the same construction applies also in the sign-changing case.

In conclusion the following result holds true.

Proposition 4.11. Let $\ell \in\{0, \ldots, m\}$ and let us assume $\alpha_{1}, \ldots, \alpha_{\ell}>0$. If $\lambda \notin \Lambda, a, b$ are regular values of $I_{\lambda}$ and all the critical points in $\left\{a \leq I_{\lambda} \leq b\right\}$ are nondegenerate, then

$$
\text { \#\{critical points of } \left.I_{\lambda} \text { in }\left\{a \leq I_{\lambda} \leq b\right\}\right\} \geq \sum_{q \geq 0} \operatorname{dim}\left(H_{q}\left(I_{\lambda}^{b}, I_{\lambda}^{a} ; \mathbb{Z}_{2}\right)\right) \text {. }
$$

\section{ON THE HOMOLOGY GROUPS OF BARYCENTER SETS}

In this section we compute the dimension of the homology groups of some spaces of formal barycenters which have been introduced in the previous section.

Keeping the notation of Proposition 4.1, we consider the space

$$
Z_{N, M}=X_{N} \amalg Y_{M},
$$

where $X_{N}=\amalg_{i=1}^{N} B^{g_{i}}$ and $Y_{M}=\left\{y_{1}, \ldots, y_{M}\right\}$, with $g_{i}$ defined in (2.8). For $k \in \mathbb{N}, N, M \in$ $\mathbb{N} \cup\{0\}$, with $N+M \geq 1$, and $q \in \mathbb{N} \cup\{0\}$ we set

$$
d_{q}(k, N, M)=\operatorname{dim}\left(\tilde{H}_{q}\left(\operatorname{Bar}_{k}\left(Z_{N, M}\right)\right) ; \mathbb{Z}_{2}\right),
$$

with the convention that $d_{q}(k, N, M)=0$ if $q<0$. 
Proposition 5.1. Let $k \in \mathbb{N}, N, M \in \mathbb{N} \cup\{0\}$, with $N+M \geq 1$, and $q \in \mathbb{N} \cup\{0\}$, then if $k+1-M \leq N$,

$$
d_{q}(k, N, M)= \begin{cases}\left(\begin{array}{l}
N+M-1 \\
N+M-p
\end{array}\right) & \sum_{\substack{a_{1}+\ldots+a_{N}=k-p+1 \\
a_{i} \geq 0}} s_{a_{1}, g_{1}} \ldots s_{a_{N}, g_{N}} \\
0 & \text { if } q=2 k-p(1 \leq p \leq k+1) \\
& \text { otherwise; }\end{cases}
$$

if $\quad k+1-M \geq N$,

$d_{q}(k, N, M)= \begin{cases}\left(\begin{array}{c}N+M-1 \\ N+M-p\end{array}\right) & \sum_{\substack{a_{1}+\ldots+a_{N}=k-p+1 \\ a_{i} \geq 0}} s_{a_{1}, g_{1}} \ldots s_{a_{N}, g_{N}} \\ \left(\begin{array}{c}N+M-s \\ M-s\end{array}\right) & \sum_{\substack{a_{1}+\ldots+a_{N}=k-N-s+1 \\ a_{i} \geq 0}} s_{a_{1}, g_{1}} \ldots s_{a_{N}, g_{N}} \\ 0 & \text { if } q=2 k-N-s \quad(1 \leq s \leq M)\end{cases}$

where $s_{a, g}=\left(\begin{array}{c}a+g-1 \\ g-1\end{array}\right)$ and $g_{i}$ is defined in (2.8).

Moreover we adopt the following convention: if $N=0$

$$
\sum_{\substack{a_{1}+\ldots+a_{N}=h \\ a_{i} \geq 0}} s_{a_{1}, g_{1}} \ldots s_{a_{N}, g_{N}}= \begin{cases}1 & \text { if } h=0 \\ 0 & \text { if } h \neq 0 .\end{cases}
$$

Notice that if $k+1-M=N$ the two formulas coincide.

Proof.

Step 1. The thesis holds true if $k=1$ or $N=0$. If $k=1, \operatorname{Bar}_{1}\left(Z_{N, M}\right) \cong Z_{N, M}$ and so by direct computation we have:

$$
d_{q}(1, N, M)= \begin{cases}\sum_{i=1}^{N} g_{i}\left(=\sum_{i=1}^{N} s_{1, g_{i}}\right) & \text { if } q=1 \\ N+M-1 & \text { if } q=0 \\ 0 & \text { otherwise. }\end{cases}
$$

If $N=0, \operatorname{Bar}_{k}\left(Z_{0, M}\right)$ is the $(k-1)$-skeleton of a $(M-1)$-symplex and so the following formula holds

$$
d_{q}(k, 0, M)= \begin{cases}\left(\begin{array}{c}
M-1 \\
k
\end{array}\right) & \text { if } q=k-1 \\
0 & \text { otherwise }\end{cases}
$$

where we adopt the convention that $\left(\begin{array}{l}a \\ b\end{array}\right)=0$ if $a<b$.

Step 2. The thesis holds true if $M=0$ for any $k \geq 2, N \geq 1$ : that is, 
$(5.2)$

$$
d_{q}(k, N, 0)= \begin{cases}\left(\begin{array}{l}
N-1 \\
N-p
\end{array}\right) & \sum_{\substack{a_{1}+\ldots+a_{N}=k-p+1 \\
a_{i} \geq 0}} s_{a_{1}, g_{1}} \ldots s_{a_{N}, g_{N}} \\
0 & \text { if } q=2 k-p(1 \leq p \leq \min \{k+1, N\}) \\
& \text { otherwise. }\end{cases}
$$

We will demonstrate (5.2) by induction on $N$, for any fixed $k \geq 2$.

If $N=0$, the formula holds by Step 1. Now, assume by induction that (5.2) holds true for a certain $N$ and let us show its validity for $N+1$. Being

$$
X_{N+1}=X_{N} \amalg B^{g_{N+1}},
$$

by Proposition 2.9, (2.11) and (2.13) we get

\section{(5.3)}

$$
\begin{aligned}
& d_{q}(k, N+1,0)=d_{q}(k, N, 0)+d_{q-1}(k-1, N, 0)+\operatorname{dim}\left(\tilde{H}_{q}\left(\operatorname{Bar}_{k}\left(B^{g_{N+1}}\right)\right)\right)+\operatorname{dim}\left(\tilde{H}_{q-1}\left(\operatorname{Bar}_{k-1}\left(B^{g_{N+1}}\right)\right)\right) \\
& +\sum_{\ell=1}^{k-1} \operatorname{dim}\left(\tilde{H}_{q}\left(\operatorname{Bar}_{k-\ell}\left(X_{N}\right) * \operatorname{Bar}_{\ell}\left(B^{g_{N+1}}\right)\right)\right)+\sum_{\ell=2}^{k-1} \operatorname{dim}\left(\tilde{H}_{q}\left(\Sigma \operatorname{Bar}_{k-\ell}\left(X_{N}\right) * \operatorname{Bar}_{\ell-1}\left(B^{g_{N+1}}\right)\right)\right),
\end{aligned}
$$

where the homology groups are intended with coefficient in $\mathbb{Z}_{2}$. Let us compute all the terms in (5.3).

The first two can be obtained using the inductive assumption. Next, again by the computations in [3, Proposition 3.2], we know that

$$
\operatorname{dim}\left(\tilde{H}_{q}\left(\operatorname{Bar}_{k}\left(B^{g_{N+1}}\right)\right)\right)= \begin{cases}s_{a_{N+1}, g_{N+1}} & \text { if } q=2 k-1 \\ 0 & \text { otherwise }\end{cases}
$$

and so

$$
\operatorname{dim}\left(\tilde{H}_{q-1}\left(\operatorname{Bar}_{k-1}\left(B^{g_{N+1}}\right)\right)\right)= \begin{cases}s_{a_{N+1}, g_{N+1}} & \text { if } q=2 k-2 \\ 0 & \text { otherwise. }\end{cases}
$$

Moreover, by (2.14), using (5.4) and the inductive assumption we have that

$$
\begin{aligned}
& \sum_{\ell=1}^{k-1} \operatorname{dim}\left(\tilde{H}_{q}\left(\operatorname{Bar}_{k-\ell}\left(X_{N}\right) * \operatorname{Bar}_{\ell}\left(B^{g_{N+1}}\right)\right)\right)= \\
& = \begin{cases}\left(\begin{array}{l}
N-1 \\
N-p
\end{array} \sum_{a_{1}+\ldots+a_{N}+\ell=k-p+1} s_{a_{i} \geq 0, \ell \geq 1} s_{a_{1}, g_{1}} \ldots s_{a_{N}, g_{N}} s_{a_{N+1}, \ell}\right. & \text { if } q=2 k-p(1 \leq p \leq N) \\
0 & \text { otherwise, }\end{cases}
\end{aligned}
$$

and 
(5.7)

$$
\begin{aligned}
& \sum_{\ell=2}^{k-1} \operatorname{dim}\left(\tilde{H}_{q}\left(\Sigma \operatorname{Bar}_{k-\ell}\left(X_{N}\right) * \operatorname{Bar}_{\ell-1}\left(B^{g_{N+1}}\right)\right)\right)= \\
& \quad= \begin{cases}\left(\begin{array}{c}
N-1 \\
N-p+1
\end{array}\right) \sum_{\substack{a_{1}+\ldots+a_{N}+\ell=k-p+1 \\
a_{i} \geq 0, \ell \geq 2}} s_{a_{1}, g_{1}} \ldots s_{a_{N}, g_{N}} s_{a_{N+1}, \ell-1} & \text { if } q=2 k-p(2 \leq p \leq N+1) \\
0 & \text { otherwise. }\end{cases}
\end{aligned}
$$

In conclusion, combining (5.3), (5.4), (5.5), (5.6) and (5.7) we obtain that

$$
d_{q}(k, N+1,0)=\left\{\begin{array}{cl}
\left(\begin{array}{c}
N \\
N+1-p
\end{array}\right) \quad \sum_{\substack{a_{1}+\ldots+a_{N+1}=k-p+1 \\
a_{i} \geq 0}} s_{a_{1}, g_{1}} \ldots s_{a_{N+1}, g_{N+1}} & \text { if } q=2 k-p(1 \leq p \leq \min \{k+1, N+1\}) \\
0 & \text { otherwise, }
\end{array}\right.
$$

so (5.2) holds true for $N+1$ and this completes the proof of (5.2).

Step 3. Conclusion.

We will prove the formula by induction on $M$, with $k \geq 2$ and $N \geq 1$ fixed.

If $M=0$ the thesis is true by Step 2. Now, let us suppose that (5.2) holds for $M$ and we prove that then it is also true for $M+1$.

Being

$$
Z_{N, M+1}=Z_{N, M} \amalg\left\{y_{M+1}\right\},
$$

and $\tilde{H}_{*}\left(\operatorname{Bar}_{k}\left(\left\{y_{M+1}\right\}\right)\right)=0$, by (2.11) and (2.13) we get

$$
d_{q}(k, N, M+1)=d_{q}(k, N, M)+d_{q-1}(k-1, N, M) .
$$

Hence by the inductive assumption we can compute $d_{q}(k, N, M+1)$, obtaining that

$$
\begin{aligned}
& \text { if } \quad k+1-(M+1) \leq N \\
& d_{q}(k, N, M+1)= \begin{cases}\left(\begin{array}{l}
N+(M+1)-1 \\
N+(M+1)-p
\end{array}\right) & \sum_{\substack{a_{1}+\ldots+a_{N}=k-p+1 \\
a_{i} \geq 0}} s_{a_{1}, g_{1} \ldots s_{a_{N}}, g_{N}} \\
0 & \text { if } q=2 k-p(1 \leq p \leq k+1) \\
0 & \text { otherwise; }\end{cases} \\
& \text { if } \quad k+1-(M+1) \geq N
\end{aligned}
$$

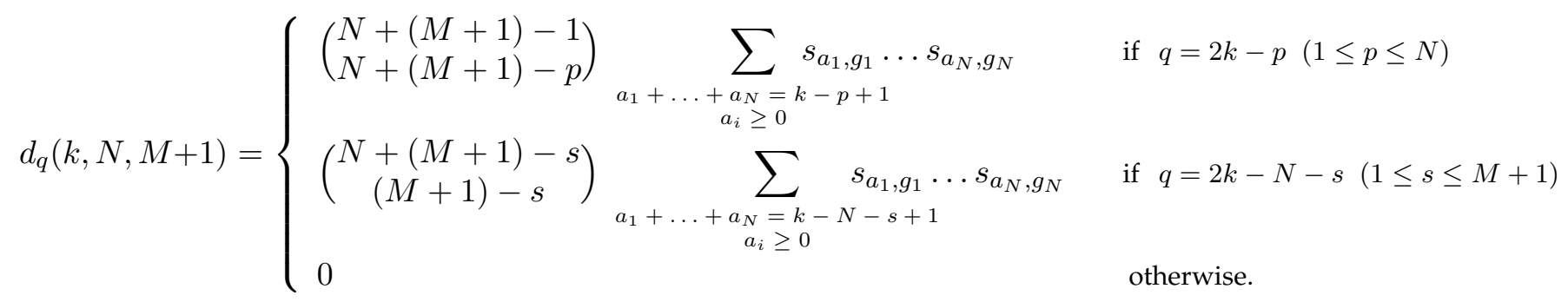


So the formula holds for $M+1$ and this concludes the proof.

Lemma 5.2. Let $N, M \in \mathbb{N} \cup\{0\}, N+M \geq 1$ and let $W_{N, M, J_{\lambda}}$ be the set defined in (4.7), then

$$
\operatorname{dim}\left(\tilde{H}_{q}\left(\operatorname{Bar}_{1}\left(W_{N, M, J_{\lambda}}\right) ; \mathbb{Z}_{2}\right)\right)= \begin{cases}N+M-1 & q=0 \\ \sum_{i=1}^{N} g_{i}+\left|J_{\lambda}\right| & q=1 \\ 0 & \text { otherwise. }\end{cases}
$$

Proof. Being $\operatorname{Bar}_{1}\left(W_{N, M, J_{\lambda}}\right) \cong W_{N, M, J_{\lambda}}$ it is immediate to see that

$$
\operatorname{dim}\left(\tilde{H}_{q}\left(W_{N, M, J_{\lambda}} ; \mathbb{Z}_{2}\right)\right)= \begin{cases}N+M-1 & q=0 \\ \sum_{i=1}^{N}\left(g_{i}+\left|J_{\lambda, A_{i}}\right|\right)+\sum_{h=1}^{M}\left|J_{\lambda, C_{h}}\right| & q=1 \\ 0 & \text { otherwise, }\end{cases}
$$

hence the thesis follows observing that

$$
\sum_{i=1}^{N}\left|J_{\lambda, A_{i}}\right|+\sum_{h=1}^{M}\left|J_{\lambda, C_{h}}\right|=\left|J_{\lambda}\right|
$$

where $J_{\lambda, A_{i}}$ and $J_{\lambda, C_{h}}$ are defined in (4.6).

\section{CONCLUSION OF THE PROOFS OF THE MAIN RESUlTS}

In order to prove our main results the following two Propositions will be of use.

Proposition 6.1. Let $\lambda \in(8 k \pi, 8(k+1) \pi), k \in \mathbb{N}$, and assume (H1), (H2).

If $b>0$ is such that $I_{\lambda}^{b}$ is contractible and $\Sigma^{+} \simeq Z_{N, M}$, where $Z_{N, M}$ is defined in (4.1), then there exists $L>0$ sufficiently large so that

$$
\operatorname{dim}\left(H_{q+1}\left(I_{\lambda}^{b}, I_{\lambda}^{-L} ; \mathbb{Z}_{2}\right)\right) \geq \operatorname{dim}\left(\tilde{H}_{q}\left(\operatorname{Bar}_{k}\left(Z_{N, M}\right) ; \mathbb{Z}_{2}\right)\right) \quad \text { for any } q \geq 0 .
$$

Proof. By assumption $I_{\lambda}^{b}$ is contractible thus, from the exactness of the homology sequence,

$$
\ldots \rightarrow \tilde{H}_{q+1}\left(I_{\lambda}^{-L} ; \mathbb{Z}_{2}\right) \rightarrow \tilde{H}_{q+1}\left(I_{\lambda}^{b} ; \mathbb{Z}_{2}\right) \rightarrow H_{q+1}\left(I_{\lambda}^{b}, I_{\lambda}^{-L} ; \mathbb{Z}_{2}\right) \rightarrow \tilde{H}_{q}\left(I_{\lambda}^{-L} ; \mathbb{Z}_{2}\right) \rightarrow \ldots
$$

we derive that

$$
\begin{aligned}
& H_{q+1}\left(I_{\lambda}^{b}, I_{\lambda}^{-L} ; \mathbb{Z}_{2}\right) \cong \tilde{H}_{q}\left(I_{\lambda}^{-L} ; \mathbb{Z}_{2}\right), \quad \text { for any } q \geq 0 \\
& H_{0}\left(I_{\lambda}^{b}, I_{\lambda}^{-L} ; \mathbb{Z}_{2}\right)=0 .
\end{aligned}
$$

Let us consider the continuous projection $\Psi$ introduced in Proposition 4.1 and the map

$$
\begin{aligned}
j: \quad \operatorname{Bar}_{k}\left(Z_{N, M}\right) & \longrightarrow I_{\lambda}^{-L} \\
\sigma=\sum_{i=1}^{k} t_{i} \delta_{x_{i}} & \mapsto \varphi_{\mu, \sigma},
\end{aligned}
$$

which is well defined by Lemma 4.3 (i) applied with $Z=Z_{N, M}$.

Then $\Psi \circ j$ is homotopically equivalent to the identity on $\operatorname{Bar}_{k}\left(Z_{N, M}\right)$. This fact follows from Proposition 4.1 and Lemma 4.3 (ii).

Hence, $\Psi_{*} \circ j_{*}=\operatorname{Id}_{\mid H_{*}\left(\operatorname{Bar}_{k}\left(Z_{N, M}\right)\right)}$ and so the desired conclusion follows by

$$
\operatorname{dim}\left(\tilde{H}_{q}\left(I_{\lambda}^{-L} ; \mathbb{Z}_{2}\right)\right) \geq \operatorname{dim}\left(\tilde{H}_{q}\left(\operatorname{Bar}_{k}\left(Z_{N, M}\right) ; \mathbb{Z}_{2}\right)\right)
$$


Proposition 6.2. Let $\lambda \in(8 \pi, 16 \pi)$ and assume (H1), (H2).

If $b>0$ is such that $I_{\lambda}^{b}$ is contractible and $\Sigma^{+} \simeq Z_{N, M}$, where $Z_{N, M}$ is defined in (4.1), then there exists $L>0$ sufficiently large so that

$$
\operatorname{dim}\left(H_{q+1}\left(I_{\lambda}^{b}, I_{\lambda}^{-L} ; \mathbb{Z}_{2}\right)\right) \geq \operatorname{dim}\left(\tilde{H}_{q}\left(\operatorname{Bar}_{1}\left(W_{N, M, J_{\lambda}}\right) ; \mathbb{Z}_{2}\right)\right) \quad \text { for any } q \geq 0,
$$

where $W_{N, M, J_{\lambda}}$ is defined in (4.7).

Proof. The proof is completely analogous to the one of the previous proposition, where $W_{N, M, J_{\lambda}}$ and $\varphi_{\mu, p, \alpha}$ play the role of $Z_{N, M}$ and $\varphi_{\mu, \sigma}$ respectively, while Proposition 4.4 and Lemma 4.6 must be applied instead of Proposition 4.1 and Lemma 4.3.

Proof of Theorem 2.2. By Lemma 4.9 there exists $b>0$ so that the sublevel $I_{\lambda}^{b}$ is contractible, then we are in position to apply Proposition 6.1 and so for $L>0$ sufficiently large

$$
\operatorname{dim}\left(H_{q+1}\left(I_{\lambda}^{b}, I_{\lambda}^{-L} ; \mathbb{Z}_{2}\right)\right) \geq \operatorname{dim}\left(\tilde{H}_{q}\left(\operatorname{Bar}_{k}\left(Z_{N, M}\right) ; \mathbb{Z}_{2}\right)\right) \quad \text { for any } q \geq 0 .
$$

Hence, by virtue of $(\mathrm{H} 3), \operatorname{dim}\left(H_{q+1}\left(I_{\lambda}^{b}, I_{\lambda}^{-L} ; \mathbb{Z}_{2}\right)\right)>0$ for some $q$, as it can be directly checked applying Theorem 5.1 and recalling that $N^{+}=N+M$. Therefore, $I_{\lambda}^{L}$ is not a retract of $I_{\lambda}^{b}$, so the conclusion follows from Lemma 4.7.

Proof of Theorem 2.3. We argue as in the proof of Theorem 2.2, indeed applying in this case Lemma 4.9 and Proposition 6.2 we have that for $b$ and $L$ sufficiently large positive

$$
\operatorname{dim}\left(H_{2}\left(I_{\lambda}^{b}, I_{\lambda}^{-L} ; \mathbb{Z}_{2}\right)\right) \geq \operatorname{dim}\left(H_{1}\left(\operatorname{Bar}_{1}\left(W_{N, M, J_{\lambda}}\right) ; \mathbb{Z}_{2}\right)\right) .
$$

Lemma 5.2 combined with $(\mathrm{H} 4)$ allows to see that $\operatorname{dim}\left(H_{2}\left(I_{\lambda}^{b}, I_{\lambda}^{-L} ; \mathbb{Z}_{2}\right)\right)>0$, so we conclude again by Lemma 4.7 .

Proof of Theorem 2.5. By virtue of Proposition 4.10, we can fix $(K, g) \in \mathcal{K}_{\ell} \times \mathcal{M}$, such that any solution $u \in I_{\lambda}^{b}$ of $(*)_{\lambda}$ is nondegenerate. Next, combining Proposition 4.11 (with $a=-L$ ) and Proposition 6.1, we get that for $L>0$ sufficiently large

$$
\begin{aligned}
\#\left\{\text { solutions to }(*)_{\lambda}\right\} & \geq \sum_{q \geq 0} \#\left\{\text { critical points in }\left\{-L \leq I_{\lambda} \leq b\right\} \text { with index } q\right\} \\
& \geq \sum_{q \geq 0} \operatorname{dim}\left(\tilde{H}_{q}\left(\operatorname{Bar}_{k}\left(Z_{N, M}\right) ; \mathbb{Z}_{2}\right)\right) .
\end{aligned}
$$

We conclude by Proposition 5.1.

Proof of Theorem 2.6. The proof is completely analogous to the one of Theorem 2.5, where $W_{N, M, J_{\lambda}}$ plays the role of $Z_{N, M}$ and Proposition 6.2 and Lemma 5.2 are applied in place of Proposition 6.1 and Proposition 5.1.

Acknowledgements. F. D. M. has been supported by PRIN 201274FYK7_005 and Fondi Avvio alla Ricerca - Sapienza 2015, whereas R .L.-S. and D. R. have been supported by the FederMineco Grant MTM2015-68210-P and by J. Andalucia (FQM116). During the preparation of this work R. L.-S. was hosted by University of Rome La Sapienza, and he wishes to thank this institution for the kind hospitality and F.D.M. for the invitation. The authors are grateful to W. Chen, C. Li and S. Kallel for their suggestions and discussions concerning the subject. Finally, 
they want to express their gratitude to the referees for their careful reading and their valuable comments on the manuscript.

\section{REFERENCES}

[1] M.O. Ahmedou, S. Kallel, C.B. Ndiaye, The resonant boundary Q-curvature problem and boundary-weighted barycenters, preprint arXiv:1604.03745.

[2] A. Ancona, Elliptic operators, conormal derivatives and positive parts of functions. With an appendix by Haïm Brezis. J. Funct. Anal. 257 (2009), no.7, 2124-2158.

[3] D. Bartolucci, F. De Marchis, A. Malchiodi, Supercritical conformal metrics with conical singularities, Int. Math. Res. Not. IMRN (2011), no. 24, 5625-5643.

[4] D. Bartolucci, C. S. Lin, Sharp existence results for mean field equations with singular data, J. Differential Equations 252 (2012), no. 7, 4115-4137.

[5] D. Bartolucci, C. S. Lin, Uniqueness results for mean field equations with singular data, Comm. Partial Differential Equations 34 (2009), no. 7-9, 676-702.

[6] D. Bartolucci, C. S. Lin, G. Tarantello, Profile of blow-up solutions to mean field equations with singular data, Comm. Partial Differential Equations 29 (2004), no. 7-8, 1241-1265.

[7] D. Bartolucci, A. Malchiodi, An improved geometric inequality via vanishing moments, with applications to singular Liouville equations, Comm. Math. Phys. 322 (2013), no. 2, 415-452.

[8] D. Bartolucci, E. Montefusco, Blow-up analysis, existence and qualitative properties of solutions for the twodimensional Emden-Fowler equation with singular potential, Math. Methods Appl. Sci. 30 (2007), no. 18, 23092327.

[9] D. Bartolucci, G. Tarantello, Liouville type equations with singular data and their applications to periodic multivortices for the electroweak theory, Comm. Math. Phys. 229 (2002), no. 1, 3-47.

[10] D. Bartolucci, G. Tarantello, Asymptotic blow-up analysis for singular Liouville type equations with applications, J. Differential Equations 262 (2017), no. 7, 3887-3931.

[11] L. Battaglia, A. Jevnikar, A. Malchiodi, D. Ruiz, A general existence result for the Toda system on compact surfaces, Adv. Math. 285 (2015), 937-979.

[12] M. Berger, Riemannian structures of prescribed Gaussian curvature for compact 2-manifolds, J. Differential Geom., 5 (1971), no. 3-4, 325-332.

[13] F. Borer, L. Galimberti, M. Struwe, "Large" conformal metrics of prescribed Gauss curvature on surfaces of higher genus, Comment. Math. Helv. 90 (2015), no. 2, 407-428.

[14] G. Bredon, Topology and geometry, volume 139 of Graduate Texts in Mathematics. Springer-Verlag, New York, 1993.

[15] H. Brezis, Functional analysis, Sobolev spaces and partial differential equations, Universitext. Springer, New York, (2011).

[16] H. Brezis, F. Merle, Uniform estimates and blow-up behavior for solutions of $-\Delta u=V(x) e^{u}$ in two dimensions, Comm. Partial Differential Equations 16 (1991), no. 8-9, 1223-1253.

[17] A. Carlotto, On the solvability of singular Liouville equations on compact surfaces of arbitrary genus, Trans. Amer. Math. Soc. 366 (2014), no. 3, 1237-1256.

[18] A. Carlotto, A. Malchiodi, Weighted barycentric sets and singular Liouville equations on compact surfaces, J. Funct. Anal. 262 (2012), no. 2, 409-450.

[19] K.C. Chang, Infinite dimensional Morse theory and multiple solution problems, PNLDE 6, Birkhäuser, Boston, 1993.

[20] W. Chen, C. Li, A priori estimates for prescribing scalar curvature equations, Ann. of Math. (2) 145 (1997), no. $3,547-564$

[21] W. Chen, C. Li, A priori estimate for the Nirenberg problem, Discrete Contin. Dyn. Syst. Ser. S 1 (2008), no. 2, 225-233.

[22] W. Chen, C. Li, Prescribing Gaussian curvatures on surfaces with conical singularities, J. Geom. Anal. 1 (1991), no.4, 359-372.

[23] W. Chen, C. Li, Moving planes, moving spheres, and a priori estimates, J. Differential Equations 195 (2003), no. 1, 1-13.

[24] C. C. Chen, C. S. Lin, Mean field equation of Liouville type with singular data: topological degree, Comm. Pure Appl. Math. 68 (2015), no. 6, 887-947. 
[25] C. C. Chen, C. S. Lin, Mean field equations of Liouville type with singular data: sharper estimates. Discrete Contin. Dyn. Syst. 28 (2010), no. 3, 1237-1272.

[26] T. D'Aprile, F. De Marchis, I. Ianni, Prescribed Gauss curvature problem on singular surfaces, preprint.

[27] F. De Marchis, Multiplicity result for a scalar field equation on compact surfaces, Comm. Partial Differential Equations 33 (2008), no. 10-12, 2208-2224.

[28] F. De Marchis, Generic multiplicity for a scalar field equation on compact surfaces, J. Funct. Anal 259 (2010), no. 8, 2165-2192.

[29] F. De Marchis, R. López-Soriano, Existence and non existence results for the singular Nirenberg problem, Calc. Var. Partial Differential Equations, 55 (2016), no. 2, paper no. 36, 35 pp.

[30] M. Del Pino, C. Román, Large conformal metrics with prescribed sign-changing Gauss curvature, Calc. Var. Partial Differential Equations 54 (2015), no. 1, 763-789.

[31] Z. Djadli, A. Malchiodi, Existence of conformal metrics with constant $Q$-curvature, Ann. of Math. 168 (2008), no. 3, 813-858.

[32] Z. Djadli, Existence result for the mean field problem on Riemann surfaces of all genuses, Commun. Contemp. Math. 10 (2008), no. 2, 205-220.

[33] G. Dunne, Self-dual Chern-Simons Theories, Lecture Notes in Physics (1995).

[34] B. Gidas, W. M. Ni, L. Nirenberg, Symmetry and Related Properties via the Maximum Principle, Comm. Math. Phys. 68 (1979), no. 3, 209-243.

[35] D. Gilbarg, N. S. Trudinger, Elliptic partial differential equations of second order, Grundlehren der Mathematischen Wissenschaften, Vol. 224. Springer-Verlag, Berlin-New York, (1977).

[36] A. Hatcher, Algebraic topology, Cambridge University Press, Cambridge, 2002.

[37] J.L. Kazdan, F. W. Warner, Curvature functions for compact 2-manifolds, Ann. of Math. (2) 99 (1974), $14-47$.

[38] T. J. Kuo, C. S. Lin, Estimates of the mean field equations with integer singular sources: non-simple blowup, J. Differential Geom. 103 (2016), no. 3, 377-424.

[39] C.H. Lai, Selected Papers on Gauge Theory of Weak and Electromagnetic Interactions, World Scientific Singapore, 1981.

[40] P. Li, R. Schoen, $L^{p}$ and mean value properties of subharmonic functions on Riemannian manifolds, Acta Math. 153 (1984), no. 3-4, 279-301.

[41] Y.Y. Li, I. Shafrir, Blow-up analysis for solutions of $-\Delta u=V e^{u}$ in dimension two, Indiana Univ. Math. J. 43 (1994), no. 4, 1255-1270.

[42] R. López-Soriano, D. Ruiz, Prescribing the Gaussian curvature in a subdomain of $\mathbb{S}^{2}$ with Neumann boundary condition, J. Geom. Anal. 26 (2016), no. 1, 630-644.

[43] M. Lucia, A mountain pass theorem without Palais-Smale condition, C. R. Math. Acad. Sci. Paris 341 (2005), no. 5, 287-291.

[44] A. Malchiodi, Morse theory and a scalar field equation on compact surfaces, Adv. Differential Equations 13 (2008), no.11-12, 1109-1129.

[45] A. Malchiodi, D. Ruiz, New improved Moser-Trudinger inequalities and singular Liouville equations on compact surfaces, Geom. Funct. Anal. 21 (2011), no. 5, 1196-1217.

[46] G. Mondello, D. Panov, Spherical metrics with conical singularities on a 2-sphere: angle constraints, Int. Math. Res. Not. IMRN 2016, no. 16, 4937-4995.

[47] J.C. Saut, R. Temam, Generic properties of nonlinear boundary value problems, Comm. Partial Differential Equations 4 (1979), no. 3, 293-319.

[48] M. Struwe, On the evolution of harmonic mappings of Riemmanian surfaces, Comment. Math. Helv. 60 (1985), no. $4,558-581$.

[49] G. Tarantello, Self-Dual Gauge Field Vortices: An Analytical Approach, PNLDE 72, Birkhäuser Boston, Inc., Boston, MA, 2007.

[50] G. Tarantello, Analytical aspects of Liouville-type equations with singular sources, Stationary partial differential equations. Vol I, 491-592, Handb. Differ. Equ., North-Holland, Amsterdam, (2004)

[51] M. Troyanov, Prescribing curvature on compact surfaces with conical singularities, Trans. Amer. Math. Soc. 324 (1991), no. 2, 793-821.

[52] Y. Yang, Solitons in Field Theory and Nonlinear Analysis, Springer-Verlag, 2001.

Francesca De Marchis, Dipartimento di Matematica, Università di Roma Sapienza, P.le Aldo Moro 5, 00185 ROMA, ITALY.

E-mail address: demarchisemat.uniroma1.it 
Rafael lópez-Soriano, Departamento de AnÁlisis Matemático, Universidad de Granada, CAmPUS FUENTENUEVA, 18071 GRANADA, SPAIN.

E-mail address: rafalseugr.es

David Ruiz, Departamento de AnÁlisis Matemático, Universidad de Granada, Campus Fuentenueva, 18071 GRANADA, SPAIN.

E-mail address: daruiz@ugr.es 